\title{
Article
}

\section{Turing instability for a attraction-repolsion chemotaxis system with logistic growth}

\author{
Abdelhakam Hassan Mohammed ${ }^{1, *}$ and Shengmao $\mathrm{Fu}^{2}$ \\ 1 Faculty of Petroleum and Hydrology Engineering, Peace University, Almugled, West Kordofan, Sudan. \\ 2 College of Mathematics and Statistics, Northwest Normal University, Lanzhou 730070, P.R. China. \\ * Correspondence: abd111hakam@gmail.com
}

Received: 17 May 2020; Accepted: 22 May 2020; Published: 22 June 2020.

Abstract: In this paper, we investigate the nonlinear dynamics for an attraction-repulsion chemotaxis Keller-Segel model with logistic source term

$$
\begin{aligned}
& u_{1 t}=d_{1} \Delta u_{1}-\chi \nabla\left(u_{1} \nabla u_{2}\right)+\xi \nabla\left(u_{1} \nabla u_{3}\right)+\mathbf{g}(u), \mathbf{x} \in \mathbb{T}^{d}, t>0, \\
& u_{2 t}=d_{2} \Delta u_{2}+\alpha u_{1}-\beta u_{2}, \mathbf{x} \in \mathbb{T}^{d}, t>0, \\
& u_{3 t}=d_{3} \Delta u_{3}+\gamma u_{1}-\eta u_{3}, \mathbf{x} \in \mathbb{T}^{d}, t>0, \\
& \frac{\partial u_{1}}{\partial x_{i}}=\frac{\partial u_{2}}{\partial x_{i}}=\frac{\partial u_{3}}{\partial x_{i}}=0, x_{i}=0, \pi, 1 \leq i \leq d, \\
& u_{1}(x, 0)=u_{10}(x), u_{2}(x, 0)=u_{20}(x), u_{3}(x, 0)=u_{30}(x), \mathbf{x} \in \mathbb{T}^{d}(d=1,2,3) .
\end{aligned}
$$

Under the assumptions of the unequal diffusion coefficients, the conditions of chemotaxis-driven instability are given in a $d$-dimensional box $\mathbb{T}^{d}=(0, \pi)^{d}(d=1,2,3)$. It is proved that in the condition of the unique positive constant equilibrium point $\mathbf{w}_{c}=\left(u_{1 c}, u_{2 c}, u_{3 c}\right)$ of above model is nonlinearly unstable. Moreover, our results provide a quantitative characterization for the early-stage pattern formation in the model.

Keywords: Attraction-repolsion chemotaxis, logistic source, pattern formation, nonlinear instability.

MSC: 35K55, 35B40, 92C17.

\section{Introduction}

I

$\mathrm{n}$ this paper, we deal with attraction-repolsion chemotaxis system

$$
\begin{cases}u_{1 t}=d_{1} \Delta u_{1}-\chi \nabla\left(u_{1} \nabla u_{2}\right)+\xi \nabla\left(u_{1} \nabla u_{3}\right)+\mathbf{g}(u), & \mathbf{x} \in \mathbb{T}^{d}, t>0, \\ u_{2 t}=d_{2} \Delta u_{2}+\alpha u_{1}-\beta u_{2}, & \mathbf{x} \in \mathbb{T}^{d}, t>0, \\ u_{3 t}=d_{3} \Delta u_{3}+\gamma u_{1}-\eta u_{3}, & \mathbf{x} \in \mathbb{T}^{d}, t>0, \\ \frac{\partial u_{1}}{\partial x_{i}}=\frac{\partial u_{2}}{\partial x_{i}}=\frac{\partial u_{3}}{\partial x_{i}}=0, & x_{i}=0, \pi, 1 \leq i \leq d, \\ u_{1}(x, 0)=u_{10}(x), u_{2}(x, 0)=u_{20}(x), u_{3}(x, 0)=u_{30}(x), & \mathbf{x} \in \mathbb{T}^{d}(d=1,2,3) .\end{cases}
$$

in a $d$-dimensional box $\mathbb{T}^{d}=(0, \pi)^{d}(d=1,2,3)$ is a bounded domain with smooth boundary $\alpha, \beta, \mu, \chi, \xi, \beta, \gamma, \eta>0$. In the model (1) $u_{1}, u_{2}$ and $u_{3}$ represent the cell density, the concentration of the chemoattractant (attractive signal) and the concentration of the chemorepellent (repulsive signal) respectively, $\mathbf{g}(u)$ is logistic source. The classical Keller-Segel system can be obtained by setting $d_{i}=1,(i=1,2,3), \xi=$ $0, u_{3} \equiv 0, \mathbf{g}(u) \equiv 0$ in (1) which models the mechanism of chemotaxis and has been extensively studied since 1970, we refer to [1-4] and the references therein. Apart form the aforementioned system a source of logistic type is included in (1) to describe the spontaneous growth of cells. The effect of preventing ultimate growth has been widely studied. 
Chemotaxis is a chemosensitive movement of species which may detect and respond to chemical substances in the environment. The first model about chemotaxis was proposed by Keller and Segel [5]

$$
\begin{cases}\frac{\partial u}{\partial t}=\Delta u-\chi \nabla(u \nabla v), & \mathbf{x} \in \Omega \\ \frac{\partial v}{\partial t}=\Delta v-v+u, & \mathbf{x} \in \Omega\end{cases}
$$

which describes the aggregation process of the slime mold formation in Dictyostelium Discoidium, where $v$ denotes the chemical concentration and $\mathrm{u}$ is the concentration of species. For this system, there have been abundant results. Osaki and Yagi [6] found that when $n=1$, all the solutions are global and bounded. When $n \geq 2$, blow-up may happen (see Horstmann and Wang [7]; Herrero et al., [8]; Winkler et al., [9]). A detailed introduction into the mathematic of the Keller-Segel model for chemotaxis is presented in Horstmann $[1,10,11]$.

In the study of chemotaxis-diffusion-growth models, the pattern dynamics is another mathematically challenging and physically important research project (see Tello and Winkler [12], Aida and Yagi [13], Kurata et al., [14], Painter and Hillen [15], Okuda and Osaki [16], Kuto et al., [17] and Banerjee et al., [18]. Guo and Hwang [19] investigated nonlinear dynamics near an unstable constant equilibrium in the classical Keller-Segel model. Their result can be interpreted as a rigorous mathematical characterization for pattern formation in the Keller-Segel model. By using the similar method, Fu and Liu [20] proved that the linear unstable positive constant equilibrium in the Keller-Segel model with a logistic source is also unstable in the full nonlinear sense. The emergence of patterns is a phenomenon frequently observed in the physical world [21].

Many authors have investigated the formation of patterns by using self-diffusive reaction-diffusion models [21-25]. Recently, some researchers made attempts to discover the effect of cross-diffusion on the pattern formation, and found that with appropriate cross-diffusion coefcients, linear reaction terms are sufficient to produce pattern formation [26-28], but there is only few attention having been paid to this direction. Therefore, based on the model (1): First, we analyse criteria of linear stability and instability of the positive constant equilibrium $\mathbf{w}_{c}$ (see Theorem 1). Second, by applying the higher-order energy estimates, the embedding theorem and the Guo-Strauss' bootstrap technique (see Guo and Strauss [29]), it is proved that for given any general perturbation of magnitude $\delta$, its nonlinear evolution is dominated by the corresponding linear dynamics along a fixed finite number of fastest growing modes, over a time period of $\ln \frac{1}{\delta}$ (see Theorem 2). We assert further that the positive constant equilibrium point $\mathbf{w}_{c}$ is nonlinearly unstable in the above conditions (Corollary 1). Each initial perturbation certainly can behave drastically differently from another, which gives rise to the richness of patterns. Our results provide a quantitative characterization for the nonlinear evolution of early-stage spatiotemporal pattern formation in the model (1).

The organization of this paper is as follows: in Section 2, we first prove Turing instability does not take place in the absence of chemotactic effect. Second, we give linear stability and instability criterions for the model (1), and discuss some properties of solutions for the corresponding linearized system. In Section 3, we consider the growing modes of (1), and prove the Bootstrap lemma. In Section 4, quantitative characterization for pattern formation and proof of nonlinear instability are given.

\section{Linear stability and instability criterions}

In this section, we study in detail linear Stability,linear instability of positive constant equilibrium point $\mathbf{w}_{c}=\left(1, \frac{\alpha}{\beta}, \frac{\gamma}{\eta}\right)$ to the model (1) in a $d$-dimensional box $\Omega=\mathbb{T}^{d}=(0, \pi)^{d}(d=1,2,3)$, and $\mathbf{g}(\mathbf{u})=\mu \mathbf{u}_{\mathbf{1}}\left(\mathbf{1}-\mathbf{u}_{1}\right)$.

\subsection{Stability of positive constant equilibrium point for (1) without chemotaxis}

We consider the stability of $\mathbf{w}_{c}$ for the corresponding system (1) without chemotaxis

$$
\begin{cases}u_{1 t}=d_{1} \Delta u_{1}+\mu u_{1}\left(1-u_{1}\right), & \mathbf{x} \in \mathbb{T}^{d}, t>0, \\ u_{2 t}=d_{2} \Delta u_{2}+\alpha u_{1}-\beta u_{2}, & \mathbf{x} \in \mathbb{T}^{d}, t>0, \\ u_{3 t}=d_{3} \Delta u_{3}+\gamma u_{1}-\eta u_{3}, & \mathbf{x} \in \mathbb{T}^{d}, t>0, \\ \frac{\partial u_{1}}{\partial x_{i}}=\frac{\partial u_{2}}{\partial x_{i}}=\frac{\partial u_{3}}{\partial x_{i}}=0, & x_{i}=0, \pi, 1 \leq i \leq d, \\ u_{1}(x, 0)=u_{10}(x), u_{2}(x, 0)=u_{20}(x), u_{3}(x, 0)=u_{30}(x), & \mathbf{x} \in \mathbb{T}^{d}(d=1,2,3) .\end{cases}
$$


For sake convenience, take $\mathbf{w}(\mathbf{x}, \mathbf{t})=\left(\mathbf{U}_{\mathbf{1}}(\mathbf{x}, \mathbf{t}), \mathbf{U}_{\mathbf{2}}(\mathbf{x}, \mathbf{t}), \mathbf{U}_{\mathbf{3}}(\mathbf{x}, \mathbf{t})\right)^{\mathbf{T}}$ and

$$
G(w)=\left(\begin{array}{c}
g_{1}(w) \\
g_{2}(w) \\
\left.g_{3}(w)\right)
\end{array}\right)=\left(\begin{array}{c}
\mu u_{1}\left(1-u_{1}\right) \\
\alpha u_{1}-\beta u_{2} \\
\left.\gamma u_{1}-\eta u_{3}\right)
\end{array}\right)
$$

then

$$
\left.\frac{\partial G}{\partial w}\right|_{w_{c}} \equiv G_{w}\left(w_{c}\right)=\left(\begin{array}{ccc}
-\mu & 0 & 0 \\
\alpha & -\beta & 0 \\
v & 0 & -\eta
\end{array}\right)
$$

Lemma 1. The positive equilibrium point $\mathbf{w}_{c}$ of (3) is locally asymptotically stable.

Proof. Let $0=k_{1}<k_{2}<k_{3}<\cdots$ be the eigenvalues of the operator $-\Delta$ on $\mathbb{T}^{d}$ with the homogeneous Neumann boundary condition, and $E\left(k_{i}\right)$ be the eigenspace corresponding to $k_{i}$ in $H^{1}\left(\mathbb{T}^{d}\right)$. Let $\mathbf{X}=\left[H^{1}\left(\mathbb{T}^{d}\right)\right]^{3}$ and $\mathbf{X}_{i j}=\left\{c \cdot \phi_{i j} \mid c \in \mathbb{R}^{3}\right\}$, where $\left\{\phi_{i j}, j=1, \cdots, \operatorname{dim} E\left(k_{i}\right)\right\}$ is an orthonormal basis of $E\left(k_{i}\right)$. Then $\mathbf{X}=\oplus_{i=1}^{\infty} \mathbf{X}_{i}$, $\mathbf{X}_{i}=\oplus_{j=1}^{\operatorname{dim} E\left(\mu_{i}\right)} \mathbf{X}_{i j}$.

Let $D=\operatorname{diag}\left(d_{1}, d_{2}, d_{3}\right)$. The linearization of (3) at $\mathbf{w}_{c}$ is

$$
\mathbf{w}_{t}=\left(D \Delta+\mathbf{G}_{\mathbf{w}}\left(\mathbf{w}_{\mathbf{c}}\right)\right) \mathbf{w} .
$$

For each $i \geq 1, \mathbf{X}_{i}$ is invariant under the operator $D \Delta+\mathbf{G}_{\mathbf{w}}\left(\mathbf{w}_{\mathbf{c}}\right)$, and $\lambda$ is an eigenvalue of this operator on $\mathbf{X}_{i}$ if and only if it is an eigenvalue of the matrix $-k_{i} D+\mathbf{G}_{\mathbf{w}}\left(\mathbf{w}_{\mathbf{c}}\right)$. The characteristic polynomial of $-k_{i} D+$ $\mathbf{G}_{\mathbf{w}}\left(\mathbf{w}_{\mathbf{c}}\right)$ is given by

$$
\operatorname{det}\left(\lambda I-\left(-k_{i} D+\mathbf{G}_{\mathbf{w}}\left(\mathbf{w}_{\mathbf{c}}\right)\right)\right)=\left(\begin{array}{ccc}
\lambda+k_{i} d_{1}+\mu & 0 & 0 \\
-\alpha & \lambda+k_{i} d_{2}+\beta & 0 \\
-\gamma & 0 & \lambda+k_{i} d_{3}+\eta
\end{array}\right)=0
$$

implies $\Psi(\lambda)=\left(\lambda+k_{i} d_{1}+\mu\right)\left(\lambda+k_{i} d_{2}+\beta\right)\left(\lambda+k_{i} d_{3}+\eta\right)=0$, then $\lambda_{1}=-\left(k_{i} d_{1}+\mu\right), \lambda_{2}=-\left(k_{i} d_{2}+\beta\right)$ and $\lambda_{3}=-\left(k_{i} d_{3}+\eta\right)$. So all the eigenvalues are negative, hence $\mathbf{w}_{c}$ is locally asymptotically stable, this complete the proof.

\subsection{Criteria of linear stability and instability}

Let $\hat{u}_{1}(\mathbf{x}, t)=u_{1}(\mathbf{x}, t)-u_{1 c}, \hat{u}_{2}(\mathbf{x}, t)=u_{2}(\mathbf{x}, t)-u_{2 c}, \hat{u}_{3}(\mathbf{x}, t)=u_{3}(\mathbf{x}, t)-u_{3 c}$ be nonlinear evolution of a perturbation around $\left(u_{1 c}, u_{2 c}, u_{3 c}\right)=\left(1, \frac{\alpha}{\beta}, \frac{v}{\eta}\right)$, and omitting the symbol " $\wedge$ ", then we rewrite (3) with

$$
\begin{cases}u_{1 t}=d_{1} \Delta u_{1}-\chi \Delta u_{2}+\xi \Delta u_{3}-\chi \nabla\left(u_{1} \nabla u_{2}\right)+\xi \nabla\left(u_{1} \nabla u_{3}\right)-\mu u_{1}\left(1+u_{1}\right), & \mathbf{x} \in \mathbb{T}^{d} \\ u_{2 t}=d_{2} \Delta u_{2}+\alpha u_{1}-\beta u_{2}, & \mathbf{x} \in \mathbb{T}^{d}, t>0, \\ u_{3 t}=d_{3} \Delta u_{3}+\gamma u_{1}-\eta u_{3}, & \mathbf{x} \in \mathbb{T}^{d}, t>0, \\ \frac{\partial u_{1}}{\partial x_{i}}=\frac{\partial u_{2}}{\partial x_{i}}=\frac{\partial u_{3}}{\partial x_{i}}=0, & x_{i}=0, \pi, 1 \leq i \leq d, \\ u_{1}(x, 0)=u_{10}(x), u_{2}(x, 0)=u_{20}(x), u_{3}(x, 0)=u_{30}(x), & \mathbf{x} \in \mathbb{T}^{d}(d=1,2,3) .\end{cases}
$$

The corresponding linearized system can be written as

$$
\begin{cases}u_{1 t}=d_{1} \Delta u_{1}-\chi \Delta u_{2}+\xi \Delta u_{3}-\mu u_{1}, & \mathbf{x} \in \mathbb{T}^{d}, t>0, \\ u_{2 t}=d_{2} \Delta u_{2}+\alpha u_{1}-\beta u_{2}, & \mathbf{x} \in \mathbb{T}^{d}, t>0, \\ u_{3 t}=d_{3} \Delta u_{3}+\gamma u_{1}-\eta u_{3}, & \mathbf{x} \in \mathbb{T}^{d}, t>0, \\ \frac{\partial u_{1}}{\partial x_{i}}=\frac{\partial u_{2}}{\partial x_{i}}=\frac{\partial u_{3}}{\partial x_{i}}=0, & x_{i}=0, \pi, 1 \leq i \leq d, \\ u_{1}(x, 0)=u_{10}(x), u_{2}(x, 0)=u_{20}(x), u_{3}(x, 0)=u_{30}(x), & \mathbf{x} \in \mathbb{T}^{d}(d=1,2,3) .\end{cases}
$$


Let $\mathbf{w}(\mathbf{x}, t) \equiv\left(u_{1}(\mathbf{x}, t), u_{2}(\mathbf{x}, t), u_{3}(\mathbf{x}, t)\right)^{\mathrm{T}}, \mathbf{q}=\left(q_{1}, \ldots, q_{d}\right) \in \mathbb{N}^{d}$ and $e_{\mathbf{q}}(\mathbf{x})=\prod_{i=1}^{d} \cos \left(q_{i} x_{i}\right)$. Then $\left\{e_{\mathbf{q}}(\mathbf{x})\right\}_{\mathbf{q} \in \mathbb{N}^{d}}$ forms a basis of the space of functions in $\mathbb{T}^{d}$ that satisfy the homogeneous Neumann boundary condition. We try to find a normal mode to the linearized system (5) of the following form

$$
\mathbf{w}(\mathbf{x}, t) \equiv \mathbf{r}_{q} e^{\lambda_{\mathbf{q}} t} e_{\mathbf{q}}(\mathbf{x}),
$$

where $\mathbf{r}_{\mathbf{q}}$ is a vector depending on $\mathbf{q}$. Substituting (6) into (5), we have

$$
\lambda_{\mathbf{q}} \mathbf{r}_{\mathbf{q}}=\left(\begin{array}{ccc}
-d_{1} q^{2}-\mu & \chi q^{2} & -\xi q^{2} \\
\alpha & -d_{2} q^{2}-\beta & 0 \\
\gamma & 0 & -d_{3} q^{2}-\eta
\end{array}\right) \mathbf{r}_{\mathbf{q}}:=\mathbf{L}_{q} \mathbf{r}_{\mathbf{q}}
$$

where $q^{2}=|\mathbf{q}|^{2}=\sum_{i=1}^{d} q_{i}^{2}$. Then the corresponding characteristic equation of $\mathbf{L}_{q}$ is

$$
\psi\left(\lambda_{\mathbf{q}}\right)=\lambda_{\mathbf{q}}^{3}+\bar{B}_{2} \lambda_{\mathbf{q}}^{2}+\bar{B}_{1} \lambda_{\mathbf{q}}+\bar{B}_{0}=0
$$

where

$$
\left\{\begin{array}{l}
\overline{B_{2}}=\left(d_{1}+d_{2}+d_{3}\right) q^{2}+(\mu+\beta+\eta):=C_{21} q^{2}+C_{22}, \\
\overline{B_{1}}=\left(d_{1} d_{2}+d_{1} d_{3}+d_{2} d_{3}\right) q^{4}+\left[\mu\left(d_{2}+d_{3}\right)+\beta\left(d_{1}+d_{3}\right)+\eta\left(d_{1}+d_{2}\right)-\alpha \chi\right. \\
-\gamma \xi] q^{2}+(\mu \beta+\mu \eta+\beta \eta):=C_{11} q^{4}+C_{12} q^{2}+C_{13}, \\
\bar{B}_{0}:=C_{01} q^{6}+C_{02} q^{4}+C_{03} q^{2}+C_{04}
\end{array}\right.
$$

and

$$
\left\{\begin{array}{l}
C_{01}:=d_{1} d_{2} d_{3} \\
C_{02}:=\beta d_{1} d_{3}+\eta d_{1} d_{3}+\mu d_{2} d_{3}-\alpha \chi d_{3}-v \xi d_{2} \\
C_{03}:=\beta d_{1} d_{2}+\eta d_{1} d_{3}+\mu d_{2} d_{3}-\eta \alpha \chi-\beta v \xi \\
C_{04}:=-\operatorname{det}\left(\mathbf{G}_{\mathbf{w}}\left(\mathbf{w}_{c}\right)\right)=\mu \beta \eta
\end{array}\right.
$$

In order to consider instability of $\mathbf{w}_{c}$, we make the following basic assumptions:

$\left(\mathrm{H}_{1}\right)$ There exists $\mathbf{q} \in \mathbb{N}^{d}$ such that the matrix $\mathbf{L}_{q}$ has at least one eigenvalue with positive real part;

$\left(\mathrm{H}_{2}\right) d_{1}, d_{2}, d_{3}>0$ and $d_{i} \neq d_{j}, i \neq j, i, j=1,2,3$.

It is know that a first necessary condition for Turing instability to happen is that $d_{i} \neq d_{j}(i \neq j)$, implying that $u_{1}, u_{2}$ and $u_{3}$ must move with different diffusion constants.

For every $\lambda_{1}(\mathbf{q}), \lambda_{2}(\mathbf{q}), \lambda_{3}(\mathbf{q})$ be the solutions of $\operatorname{det}\left(\lambda_{\mathbf{q}} I-\mathbf{L}_{q}\right)=0$. It will be state by Lemma 3 that there exist finitely many values $\mathbf{q} \in \mathbb{N}^{d}$ such that

$$
\max \left\{\operatorname{Re} \lambda_{1}(\mathbf{q}), \operatorname{Re} \lambda_{2}(\mathbf{q}), \operatorname{Re} \lambda_{3}(\mathbf{q})\right\}>0 .
$$

Hence there exists one $q^{2}$ having the largest eigenvalue

$$
\lambda_{\max }=\max _{\mathbf{q} \in \mathbb{N}^{d}} \max _{1 \leq i \leq 3} \operatorname{Re} \lambda_{i}\left(q^{2}\right)>0 .
$$

$\left(\mathrm{H}_{3}\right)$ At $q=\left(\bar{q}_{1}, \cdots, \bar{q}_{d}\right) \in \mathbb{N}^{d}$ which attains $\lambda_{\max }=\operatorname{Re} \lambda_{i}(q)$, we assume that the Jordan canonical form of the matrix $\mathbf{L}_{\bar{q}}=\mathbf{G}_{\mathbf{w}}\left(\mathbf{w}_{c}\right)+\mathbf{Q}\left(\bar{q}^{2}\right)$ is $J=\operatorname{diag}\left(\lambda_{1}(q), \lambda_{2}(q), \lambda_{3}(q)\right)$, where $\bar{q}^{2}=\sum_{i=1}^{d} \bar{q}_{i}^{2}$ and

$$
\mathbf{Q}\left(\bar{q}^{2}\right):=\left(\begin{array}{ccc}
-d_{1} \bar{q}^{2} & \chi \bar{q}^{2} & \xi \bar{q}^{2} \\
0 & -d_{2} \bar{q}^{2} & 0 \\
0 & 0 & -d_{3} \bar{q}^{2}
\end{array}\right)
$$


Let us carry on discussion on the characteristic equation (7). Denote

$$
A:=\bar{B}_{2}^{2}-3 \bar{B}_{1}, B:=\bar{B}_{2} \bar{B}_{1}-9 \bar{B}_{0}, C:=\bar{B}_{1}^{2}-3 \bar{B}_{2} \bar{B}_{0}
$$

and

$$
\begin{aligned}
\Delta & =B^{2}-4 A C=3\left\{4 \bar{B}_{1}^{3}+4 \bar{B}_{2}^{3} \bar{B}_{0}+27 \bar{B}_{0}^{2}-\bar{B}_{2}^{2} \bar{B}_{1}^{2}-18 \bar{B}_{2} \bar{B}_{1} \bar{B}_{0}\right\} \\
& :=Q_{6} q^{12}+Q_{5} q^{10}+Q_{4} q^{8}+Q_{3} q^{6}+Q_{2} q^{4}+Q_{1} q^{2}+Q_{0},
\end{aligned}
$$

where

$$
\begin{aligned}
Q_{6}= & 3\left\{4 C_{21}^{3} C_{01}+27 C_{01}-C_{21}^{2} C_{11}^{2}-18 C_{21} C_{11} C_{01}\right\}, \\
Q_{5}= & 6\left\{27 C_{01} C_{02}+2 C_{21}^{3} C_{02}+6 C_{21}^{2} C_{22} C_{01}-C_{21}^{2} C_{11} C_{12}-C_{21} C_{11}^{2} C_{22}\right. \\
& \left.-9 C_{21} C_{11} C_{02}-9 C_{21} C_{12} C_{01}-9 C_{22} C_{11} C_{01}\right\} \\
Q_{4}= & 3\left\{27 C_{02}+54 C_{01} C_{03}+4 C_{21}^{2} C_{03}+12 C_{21}^{2} C_{22} C_{02}+12 C_{21} C_{22}^{2} C_{01}+4 C_{11}^{2}\right. \\
& -C_{21}^{2} C_{12}^{2}-2 C_{11} C_{13} C_{21}^{2}-4 C_{21} C_{22} C_{11} C_{03}-C_{22}^{2} C_{11}^{2}-18 C_{21} C_{11} C_{03} \\
& \left.-18 C_{21} C_{12} C_{02}-18 C_{21} C_{13} C_{01}-18 C_{22} C_{11} C_{02}-18 C_{22} C_{12} C_{01}\right\}, \\
Q_{3}= & 6\left\{27 C_{01} C_{04}+27 C_{02} C_{03}+2 C_{21}^{3} C_{04}+12 C_{22}^{3} C_{01}+6 C_{21}^{2} C_{22} C_{03}\right. \\
& +6 C_{21} C_{22}^{2} C_{02}+4 C_{11} C_{12}-C_{21}^{2} C_{12} C_{13}-C_{21} C_{22} C_{12}^{2}-2 C_{21} C_{22} C_{11} C_{13} \\
& -C_{22}^{2} C_{11} C_{12}-9 C_{21} C_{11} C_{04}-9 C_{21} C_{12} C_{03}-9 C_{21} C_{13} C_{02} \\
& \left.-9 C_{22} C_{11} C_{03}-9 C_{22} C_{12} C_{02}-9 C_{22} C_{13} C_{01}\right\} \\
Q_{2}= & 3\left\{27 C_{03}+54 C_{02} C_{04}+4 C_{22}^{3} C_{02}+12 C_{21}^{2} C_{22} C_{04}+4 C_{12}^{2}+12 C_{21} C_{22}^{2} C_{03}\right. \\
& +8 C_{11} C_{13}-C_{21}^{2} C_{13}^{2}-4 C_{21} C_{22} C_{12} C_{13}-C_{22}^{2} C_{12}^{2}-2 C_{22}^{2} C_{11} C_{13}-18 C_{21} C_{12} C_{04} \\
& \left.-18 C_{21} C_{13} C_{03}-18 C_{22} C_{11} C_{04}-18 C_{22} C_{13} C_{02}-18 C_{22} C_{12} C_{03}\right\}, \\
Q_{1}= & 6\left\{27 C_{03} C_{04}+2 C_{22}^{3} C_{03}+6 C_{21} C_{22}^{2} C_{04}+4 C_{12} C_{13}-C_{21} C_{22} C_{13}^{2}-C_{12} C_{13} C_{22}^{2}\right. \\
& \left.-9 C_{21} C_{13} C_{04}-9 C_{22} C_{12} C_{04}-9 C_{22} C_{13} C_{03}\right\} \\
Q_{0}= & 3\left\{4 C_{22}^{3} C_{04}+4 C_{13}^{2}+27 C_{04}^{2}-C_{22}^{2} C_{13}^{2}-18 C_{22} C_{13} C_{04}\right\} .
\end{aligned}
$$
follows

The derivative of $\psi\left(\lambda_{\mathbf{q}}\right)$ is $\psi^{\prime}\left(\lambda_{\mathbf{q}}\right)=3 \lambda_{\mathbf{q}}^{2}+2 \bar{B}_{2} \lambda_{\mathbf{q}}+\bar{B}_{1}$. Obviously, equation $\psi^{\prime}\left(\lambda_{\mathbf{q}}\right)=0$ has two roots as

$$
\begin{aligned}
\lambda_{1,2}^{*}(\mathbf{q}) Z & =\frac{1}{3}\left(-\bar{B}_{2} \pm \sqrt{\bar{B}_{2}^{2}-3 \bar{B}_{1}}\right) \\
& =\frac{1}{3}\left[-\left(C_{21} q^{2}+C_{22} \pm \sqrt{\left(C_{21}^{2}-3 C_{11}\right) q^{4}+\left(2 C_{21} C_{22}-3 C_{12}\right) q^{2}+\left(C_{22}^{2}-3 C_{13}\right)}\right]\right. \\
& =\frac{1}{3}\left[-\left(C_{21} q^{2}+C_{22}\right) \pm \sqrt{\left(C_{21} q^{2}+C_{22}\right)^{2}-3\left(C_{11} q^{4}+C_{12} q^{2}+C_{13}\right)}\right] .
\end{aligned}
$$

Next, let us give one result concerning the cubic equation in Hu et al., [30] (which was first introduced in Fan [31]), which is used to discuss the linear stability and instability of positive constant equilibrium solution for the model (1).

Lemma 2. Let equation $x^{3}+b x^{2}+c x+d=0$, where $b, c, d \in \mathbb{R}$. Let further $A=b^{2}-3 c, B=b c-9 d, C=$ $c^{2}-3 b d$ and $\Delta=B^{2}-4 A C$. Then the equation has three real roots if and only if $\Delta \leq 0$; the equation has one real root and a pair of conjugate complex roots if and only if $\Delta>0$. Furthermore, the conjugate complex roots are $w=\frac{-2 b+Y_{1}^{1 / 3}+Y_{2}^{1 / 3}}{6} \pm i \frac{\sqrt{3}\left(Y_{1}^{1 / 3}-Y_{2}^{1 / 3}\right)}{6}$, where $Y_{1,2}=b A+\frac{3\left(-B \pm \sqrt{B^{2}-4 A C}\right)}{2}$. 
According to Lemma 2, on the one hand, if $\Delta \leq 0$, then (7) has three real roots $\lambda_{1}(\mathbf{q}), \lambda_{2}(\mathbf{q}), \lambda_{3}(\mathbf{q})$, and denote $\lambda_{1}(\mathbf{q}) \leq \lambda_{2}(\mathbf{q}) \leq \lambda_{3}(\mathbf{q})$. From this, we further infer that $\lambda_{1,2}^{*}(\mathbf{q})$ also are real. Moreover, recall that $\bar{B}_{2}=-\left(\lambda_{1}(\mathbf{q})+\lambda_{2}(\mathbf{q})+\lambda_{3}(\mathbf{q})\right)>0$, it means that (7) has at least one eigenvalue with negative real part. On the other hand, if $\Delta>0$, then Equation (7) has one real root $\lambda_{1}(\mathbf{q})$ and a pair of conjugate complex roots

$$
\lambda_{2,3}(\mathbf{q})=\frac{-2 \bar{B}_{2}+Y_{1}^{1 / 3}+Y_{2}^{1 / 3}}{6} \pm \mathrm{i} \frac{\sqrt{3}\left(Y_{1}^{1 / 3}-Y_{2}^{1 / 3}\right)}{6}
$$

with

$$
Y_{1,2}=\bar{B}_{2} A+\frac{3\left(-B \pm \sqrt{B^{2}-4 A C}\right)}{2} .
$$

Notice by the Routh-Hurwitz criterion that $\mathbf{q}=0$, in the case of $C_{22} C_{13}>C_{04}$, then (8) has three negative roots. So we consider the case $\mathbf{q} \neq 0$ in the sequel.

In this section, our first main purpose is to give criteria for linear stability and instability of $\mathbf{w}_{c}$.

Theorem 1. (Linear stability and instability). Let $\mathbf{w}_{c}$ be positive constant equilibrium solution of (1). Assume that $\lambda_{1}, \lambda_{2}$ and $\lambda_{3}$ are three roots of $\psi(\lambda)=\lambda^{3}+\bar{B}_{2} \lambda^{2}+\bar{B}_{1} \lambda+\bar{B}_{0}=0$, and that $\lambda_{1}^{*}$ and $\lambda_{2}^{*}$ are two roots of $\psi^{\prime}(\lambda)=$ $3 \lambda^{2}+2 \bar{B}_{2} \lambda+\bar{B}_{1}=0$, then we have the following conclusions:

(1) If one of the following conditions holds, then $\mathbf{w}_{c}$ is linearly stable.

$\left(\boldsymbol{H}_{S 1}\right) \Delta \leq 0, \bar{B}_{0}>0$ and $\lambda_{1}^{*}<\lambda_{2}^{*}<0$.

$\left(H_{S 2}\right) \Delta>0, \bar{B}_{0}>0$ and the conjugate complex roots $\lambda_{2}, \lambda_{3}$ satisfy $\operatorname{Re} \lambda_{2}<0, \operatorname{Re} \lambda_{3}<0$.

(2) If one of the following conditions holds, then $\mathbf{w}_{c}$ is linearly unstable.

$\left(H_{U 1}\right) \Delta \leq 0$, and one of the following conditions holds:

$\left(\boldsymbol{H}_{U 11}\right) \bar{B}_{0}>0$ and $\lambda_{2}^{*}>\lambda_{1}^{*}>0$.

( $\left.\boldsymbol{H}_{U 12}\right) \bar{B}_{0}>0$ and $\lambda_{2}^{*}>0>\lambda_{1}^{*}$.

( $\left.\boldsymbol{H}_{U 13}\right) \bar{B}_{0}<0$ and $\lambda_{2}^{*}>0>\lambda_{1}^{*}$.

$\left(\boldsymbol{H}_{U 14}\right) \bar{B}_{0}<0$ and $\lambda_{1}^{*}<\lambda_{2}^{*}<0$.

$\left(\boldsymbol{H}_{U 2}\right) \Delta>0$, and one of the following conditions holds:

( $\left.H_{U 21}\right) \bar{B}_{0}>0$ and the conjugate complex roots $\lambda_{2}, \lambda_{3}$ satisfy $\operatorname{Re} \lambda_{2}>0, \operatorname{Re} \lambda_{3}>0$.

$\left(H_{U 22}\right) \bar{B}_{0}<0$ and the conjugate complex roots $\lambda_{2}, \lambda_{3}$ satisfy $\operatorname{Re} \lambda_{2}<0, \operatorname{Re} \lambda_{3}<0$.

Here $\Delta=B^{2}-4 A C, A:=\bar{B}_{2}^{2}-3 \bar{B}_{1}, B:=\bar{B}_{2} \bar{B}_{1}-9 \bar{B}_{0}, C:=\bar{B}_{1}^{2}-3 \bar{B}_{2} \bar{B}_{0}$, in particular, $\bar{B}_{0}=\psi(0)=$ $-\lambda_{1} \lambda_{2} \lambda_{3}$

Proof. Let $\Delta \leq 0$. By Lemma 2, the equation $\psi(\lambda)=\lambda^{3}+\bar{B}_{2} \lambda^{2}+\bar{B}_{1} \lambda+\bar{B}_{0}=0$ has three real roots $\lambda_{1}, \lambda_{2}$ and $\lambda_{3}$ and assume $\lambda_{1} \leq \lambda_{2} \leq \lambda_{3}$. Moreover, the equation $\psi^{\prime}(\lambda)=3 \lambda^{2}+2 \bar{B}_{2} \lambda+\bar{B}_{1}=0$ has also two real roots $\lambda_{1}^{*}$ and $\lambda_{2}^{*}$ with $\lambda_{1}^{*} \leq \lambda_{2}^{*}$, and

$$
\begin{aligned}
& \psi^{\prime}(\lambda)>0, \forall \lambda \in\left(-\infty, \lambda_{1}^{*}\right) \cup\left(\lambda_{2}^{*},+\infty\right), \\
& \psi^{\prime}(\lambda)<0, \forall \lambda \in\left(\lambda_{1}^{*}, \lambda_{2}^{*}\right) .
\end{aligned}
$$

Therefore,

$$
\psi\left(\lambda_{1}^{*}\right) \geq 0, \psi\left(\lambda_{2}^{*}\right) \leq 0
$$

and

$$
\lambda_{1} \in\left(-\infty, \lambda_{1}^{*}\right], \lambda_{2} \in\left[\lambda_{1}^{*}, \lambda_{2}^{*}\right], \lambda_{3} \in\left[\lambda_{2}^{*},+\infty\right) .
$$

Let condition $\left(\mathbf{H}_{U 11}\right)$ hold. If $\lambda_{1}^{*}>0$, then $\lambda_{2}>0, \lambda_{3}>0$. Since $\psi(\lambda)$ is increasing for all $\lambda \in\left(-\infty, \lambda_{1}^{*}\right]$ and $\psi(0)=\bar{B}_{0}>0$, one has $\lambda_{1}<0$. If $\lambda_{1}>0$, this contradicts $\bar{B}_{2}>0$. Hence, $\mathbf{w}_{c}$ is linearly unstable.

Under the condition $\left(\mathbf{H}_{U 12}\right)$, if $\lambda_{1}^{*}<0, \lambda_{2}^{*}>0$, then $\lambda_{1}<0$. Since $\psi(\lambda)$ is decreasing for all $\lambda \in\left(\lambda_{1}^{*}, \lambda_{2}^{*}\right)$ and $\bar{B}_{0}>0$, we have $\lambda_{2}>0, \lambda_{3}>0$. This means that $\mathbf{w}_{c}$ is linearly unstable.

Similarly, it is proved that when condition $\left(\mathbf{H}_{U 13}\right)$ or $\left(\mathbf{H}_{U 14}\right)$ holds, eigenvalues $\lambda_{1}<0, \lambda_{2}<0$ and $\lambda_{3}>0$, that is, $\mathbf{w}_{c}$ is linearly unstable.

In the case $\left(\mathbf{H}_{S 1}\right)$, By monotonicity of $\psi(\lambda)$ for all $\lambda \in\left(\lambda_{2}^{*},+\infty\right)$, it holds $\lambda_{1}<0, \lambda_{2}<0$ and $\lambda_{3}<0$. Hence, $\mathbf{w}_{c}$ is linearly stable. 
We now let $\Delta>0$. In view of Lemma $2, \psi(\lambda)=0$ has one real root $\lambda_{1}$ and a pair of conjugate complex roots $\lambda_{2}, \lambda_{3}$. If condition $\left(\mathbf{H}_{U 21}\right)$ holds, then it follows from $\bar{B}_{0}>0$ that real root $\lambda_{1}<0$. Therefore, $\mathbf{w}_{c}$ is linearly unstable based on $\operatorname{Re} \lambda_{2}>0, \operatorname{Re} \lambda_{3}>0$. Similarly, we can also prove that if condition $\left(\mathbf{H}_{U 22}\right)$ holds, then $\mathbf{w}_{c}$ is linearly unstable. If condition $\left(\mathbf{H}_{S 2}\right)$ holds, it is easily to obtain that $\mathbf{w}_{c}$ is linearly stable. This completes the proof.

\subsection{Some properties of solutions of the linearized system (5)}

Lemma 3. If $\mathbf{q} \in \mathbb{N}^{d}$ and $q^{2}$ sufficiently large, then all eigenvalues of $\boldsymbol{L}_{q}$ have negative real parts.

Proof. Notice that $C_{21}, C_{22}, C_{11}, C_{13}, C_{01}, C_{04}$ and $\bar{B}_{2}$ are all positive, where the parameters are mentioned in (8) and (9). In addition, $\bar{B}_{2}, \bar{B}_{1}, \bar{B}_{0}$ and $\bar{B}_{2} \bar{B}_{1}-\bar{B}_{0}$ are positive if $\mathbf{q} \in \mathbb{N}^{d}$ sufficiently large. It is follows from the Routh-Hurwitz criterion that all eigenvalues of $\mathbf{L}_{q}$ have negative real parts for $\mathbf{q} \in \mathbb{N}^{d}$ sufficiently large.

For given $\mathbf{q} \in \mathbb{N}^{d}$, let $\lambda_{1}(\mathbf{q}), \lambda_{2}(\mathbf{q}), \lambda_{3}(\mathbf{q})$ be the eigenvalues of $\mathbf{L}_{q}$ and the corresponding eigenvectors by $\mathbf{r}_{1}(\mathbf{q}), \mathbf{r}_{2}(\mathbf{q}), \mathbf{r}_{3}(\mathbf{q})$. According to eigenvectors, we divide $\mathbf{q}$ into the following four cases to analyze:

Case 1: $\mathbf{q} \in \mathbb{N}_{R 1}^{d}$ :

$\mathbf{L}_{q}$ has three real eigenvalues $\lambda_{1}(\mathbf{q}), \lambda_{2}(\mathbf{q})$ and $\lambda_{3}(\mathbf{q})$, and three corresponding linearly independent eigenvectors $\mathbf{r}_{1}(\mathbf{q}), \mathbf{r}_{2}(\mathbf{q})$ and $\mathbf{r}_{3}(\mathbf{q})$. In the case we arrange $\lambda_{1}(\mathbf{q}) \leq \lambda_{2}(\mathbf{q}) \leq \lambda_{3}(\mathbf{q})$.

\section{Case 2: $\mathbf{q} \in \mathbb{N}_{R 2}^{d}$ :}

$\mathbf{L}_{q}$ has a single root $\lambda_{1}(\mathbf{q})=\lambda_{s}(\mathbf{q})$ and a double root $\lambda_{2}(\mathbf{q})=\lambda_{3}(\mathbf{q})=\lambda_{d}(\mathbf{q})$ (or $\mathbf{L}_{q}$ has three repeated real root $\lambda_{s}(\mathbf{q})=\lambda_{d}(\mathbf{q})$ ), meanwhile, there are only two linearly independent real eigenvectors $\mathbf{r}_{s}(\mathbf{q})$ and $\mathbf{r}_{d}(\mathbf{q})$. In this case we need find another independent vector $\mathbf{r}_{d}^{\prime}(\mathbf{q})$ satisfying

$$
\left(\mathbf{L}_{q}-\lambda_{d}(\mathbf{q}) \mathrm{I}\right) \mathbf{r}_{d}^{\prime}(\mathbf{q})=\mathbf{r}_{d}(\mathbf{q})
$$

Case 3: $\mathbf{q} \in \mathbb{N}_{R 3}^{d}$ :

(7) has a triple eigenvalue $\lambda(\mathbf{q})$ which only corresponding one linearly independent eigenvector $\mathbf{r}(\mathbf{q})$. In this case, we need to supplement another two independent vectors $\mathbf{r}^{\prime}(\mathbf{q})$ and $\mathbf{r}^{\prime \prime}(\mathbf{q})$, which satisfy

$$
\left(\mathbf{L}_{q}-\lambda(\mathbf{q}) \mathrm{I}\right) \mathbf{r}^{\prime}(\mathbf{q})=\mathbf{r}(\mathbf{q}),\left(\mathbf{L}_{q}-\lambda(\mathbf{q}) \mathrm{I}\right) \mathbf{r}^{\prime \prime}(\mathbf{q})=\mathbf{r}^{\prime}(\mathbf{q}) .
$$

Case 4: $\mathbf{q} \in \mathbb{N}_{C}^{d}=\mathbb{N}^{d}-\left(\mathbb{N}_{R 1}^{d} \cup \mathbb{N}_{R 2}^{d} \cup \mathbb{N}_{R 3}^{d}\right)$ :

The characteristic equation (7) has one real root and a pair of conjugate complex roots. The eigenvalues and the corresponding eigenvectors are denoted by $\lambda_{r}(\mathbf{q}), \operatorname{Re} \lambda_{c}(\mathbf{q})+i \operatorname{Im} \lambda_{c}(\mathbf{q}), \operatorname{Re} \lambda_{c}(\mathbf{q})-i \operatorname{Im} \lambda_{c}(\mathbf{q})$ and $\mathbf{r}(\mathbf{q})$, $\operatorname{Rer}_{c}(\mathbf{q})+i \operatorname{Im} \mathbf{r}_{c}(\mathbf{q}), \operatorname{Rer}_{c}(\mathbf{q})-i \operatorname{Im} \mathbf{r}_{c}(\mathbf{q})$, respectively. Notice that $\operatorname{Rer}_{c}(\mathbf{q})$ and $\operatorname{Im}_{c}(\mathbf{q})$ are linearly independent vectors.

Given any initial perturbation $\mathbf{w}(\mathbf{x}, 0)$, it can be expressed as

$$
\begin{aligned}
\mathbf{w}(\mathbf{x}, 0)= & \mathbf{w}_{0}(\mathbf{x})=\sum_{\mathbf{q} \in \mathbb{N}^{d}} \mathbf{w}_{\mathbf{q}} e_{\mathbf{q}}(\mathbf{x}) \\
= & \sum_{\mathbf{q} \in \mathbb{N}_{R 1}^{d}}\left[w_{1}(\mathbf{q}) \mathbf{r}_{1}(\mathbf{q})+w_{2}(\mathbf{q}) \mathbf{r}_{2}(\mathbf{q})+w_{3}(\mathbf{q}) \mathbf{r}_{3}(\mathbf{q})\right] e_{\mathbf{q}}(\mathbf{x}) \\
& +\sum_{\mathbf{q} \in \mathbb{N}_{R 2}^{d}}\left[w_{d}(\mathbf{q}) \mathbf{r}_{d}(\mathbf{q})+w_{d}^{\prime}(\mathbf{q}) \mathbf{r}_{d}^{\prime}(\mathbf{q})+w_{\mathcal{S}}(\mathbf{q}) \mathbf{r}_{s}(\mathbf{q})\right] e_{\mathbf{q}}(\mathbf{x}) \\
& +\sum_{\mathbf{q} \in \mathbb{N}_{R 3}^{d}}\left[w(\mathbf{q}) \mathbf{r}(\mathbf{q})+w^{\prime}(\mathbf{q}) \mathbf{r}^{\prime}(\mathbf{q})+w^{\prime \prime}(\mathbf{q}) \mathbf{r}^{\prime \prime}(\mathbf{q})\right] e_{\mathbf{q}}(\mathbf{x}) \\
& +\sum_{\mathbf{q} \in \mathbb{N}_{C}^{d}}\left[w^{\operatorname{Re}}(\mathbf{q}) \operatorname{Rer}_{c}(\mathbf{q})+w^{\operatorname{Im}}(\mathbf{q}) \operatorname{Im}_{c}(\mathbf{q})+w_{r}(\mathbf{q}) \mathbf{r}_{r}(\mathbf{q})\right] e_{\mathbf{q}}(\mathbf{x}),
\end{aligned}
$$

where $w_{i}(\mathbf{q}), w_{d}(\mathbf{q}), w_{d}^{\prime}(\mathbf{q}), w_{s}(\mathbf{q}), w(\mathbf{q}), w^{\prime}(\mathbf{q}), w^{\prime \prime}(\mathbf{q}), w^{\operatorname{Re}}(\mathbf{q}), w^{\operatorname{Im}}(\mathbf{q}), w_{r}(\mathbf{q}) \in \mathbb{R}, i=1,2,3$ and 


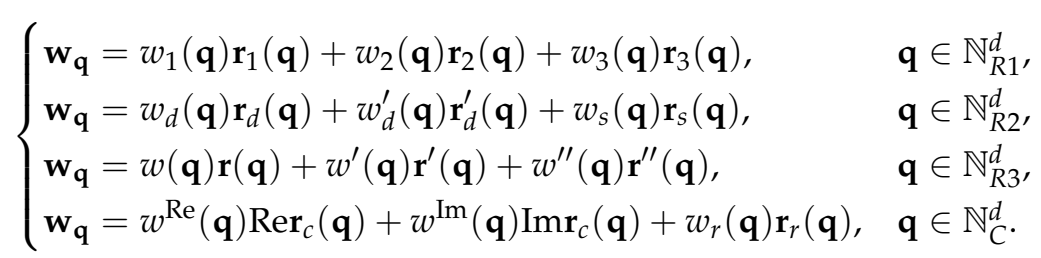

Thus, the unique solution $\mathbf{w}(\mathbf{x}, t)$ to the linearized system (5) can be written in the following form.

$$
\begin{aligned}
\mathbf{w}(\mathbf{x}, t)= & \sum_{\mathbf{q} \in \mathbb{N}_{R 1}^{d}}\left[w_{1}(\mathbf{q}) \mathbf{r}_{1}(\mathbf{q}) e^{\lambda_{1}(\mathbf{q}) t}+w_{2}(\mathbf{q}) \mathbf{r}_{2}(\mathbf{q}) e^{\lambda_{2}(\mathbf{q}) t}+w_{3}(\mathbf{q}) \mathbf{r}_{3}(\mathbf{q}) e^{\lambda_{3}(\mathbf{q}) t}\right] e_{\mathbf{q}}(\mathbf{x}) \\
& +\sum_{\mathbf{q} \in \mathbb{N}_{R 2}^{d}}\left\{\left[w_{d}(\mathbf{q}) \mathbf{r}_{d}(\mathbf{q})+w_{d}^{\prime}(\mathbf{q})\left(\mathbf{r}_{d}^{\prime}(\mathbf{q})+\mathbf{r}_{d}(\mathbf{q}) t\right)\right] e^{\lambda_{d}(\mathbf{q}) t}+w_{s}(\mathbf{q}) \mathbf{r}_{s}(\mathbf{q}) e^{\lambda_{s}(\mathbf{q}) t}\right\} e_{\mathbf{q}}(\mathbf{x}) \\
& +\sum_{\mathbf{q} \in \mathbb{N}_{R 3}^{d}}\left[w(\mathbf{q}) \mathbf{r}(\mathbf{q})+w^{\prime}(\mathbf{q})\left(\mathbf{r}^{\prime}(\mathbf{q})+\mathbf{r}(\mathbf{q}) t\right)+w^{\prime \prime}(\mathbf{q})\left(\mathbf{r}^{\prime \prime}(\mathbf{q})+\mathbf{r}^{\prime}(\mathbf{q}) t+\mathbf{r}(\mathbf{q}) t^{2}\right)\right] \\
& \times e^{\lambda(\mathbf{q}) t} e_{\mathbf{q}}(\mathbf{x})+\sum_{\mathbf{q} \in \mathbb{N}_{C}^{d}}\left\{\left[w^{\operatorname{Re}}(\mathbf{q})\left(\operatorname{Rer}_{c}(\mathbf{q}) \cos \left[\left(\operatorname{Im} \lambda_{c}(\mathbf{q})\right) t\right]-\operatorname{Im} \mathbf{r}_{c}(\mathbf{q}) \sin \left[\left(\operatorname{Im} \lambda_{c}(\mathbf{q})\right) t\right]\right)\right.\right. \\
& \left.+w^{\operatorname{Im}}(\mathbf{q})\left(\operatorname{Rer}_{c}(\mathbf{q}) \sin \left[\left(\operatorname{Im} \lambda_{c}(\mathbf{q})\right) t\right]+\operatorname{Im} \mathbf{r}_{c}(\mathbf{q}) \cos \left[\left(\operatorname{Im} \lambda_{c}(\mathbf{q})\right) t\right]\right)\right] e^{\left(\operatorname{Re} \lambda_{c}(\mathbf{q})\right) t} \\
& \left.+w_{r}(\mathbf{q}) \mathbf{r}_{r}(\mathbf{q}) e^{\lambda_{r}(\mathbf{q}) t}\right\} e_{\mathbf{q}}(\mathbf{x}) \\
:= & \sum_{\mathbf{q} \in \mathbb{N}_{R 1}^{d}} T_{R 1}\left(\mathbf{w}_{q}\right)(\mathbf{x}, t)+\sum_{\mathbf{q} \in \mathbb{N}_{R 2}^{d}} T_{R 2}\left(\mathbf{w}_{q}\right)(\mathbf{x}, t)+\sum_{\mathbf{q} \in \mathbb{N}_{R 3}^{d}} T_{R 3}\left(\mathbf{w}_{q}\right)(\mathbf{x}, t)+\sum_{\mathbf{q} \in \mathbb{N}_{C}^{d}} T_{\mathcal{c}}\left(\mathbf{w}_{q}\right)(\mathbf{x}, t) \\
\equiv & e^{\mathfrak{L} t} \mathbf{w}_{0}(\mathbf{x}) .
\end{aligned}
$$

Recall that

$$
\lambda_{\max }=\max _{\mathbf{q} \in \mathbb{N}^{d}} \max _{1 \leq i \leq 3} \operatorname{Re} \lambda_{i}(\mathbf{q})>0,
$$

where $\lambda_{1}(\mathbf{q}), \lambda_{2}(\mathbf{q}), \lambda_{3}(\mathbf{q})$ are the solutions of (7). Denote

$$
\mathbb{N}_{\text {max }}^{d}=\left\{\mathbf{q} \in \mathbb{N}^{d} \mid \operatorname{Re} \lambda_{i}(\mathbf{q})=\lambda_{\max }, i=1,2,3\right\}
$$
$\mathbb{N}_{C}^{d}$.

By the assumption $\left(\mathbf{H}_{3}\right)$, the largest eigenvalue $\lambda_{\max }$ can be obtained, provided that $\mathbf{q}$ belongs to $\mathbb{N}_{R 1}^{d}$ or In the sequel, we define

$$
I=\{i \mid 1 \leq i \leq 3\}, I_{1}=\left\{i \mid \lambda_{i}(\mathbf{q})=\lambda_{\max }, 1 \leq i \leq 3\right\},
$$

and

$$
\begin{aligned}
& \Lambda_{R 1}=\mathbb{N}_{R 1}^{d} \cap \mathbb{N}^{d}{ }_{\max }, \Lambda_{C}=\mathbb{N}_{C}^{d} \cap \mathbb{N}^{d}{ }_{\max }, \\
& \Lambda_{C 1}=\left\{\mathbf{q} \in \Lambda_{C} \mid \operatorname{Re} \lambda_{C}(\mathbf{q})=\lambda_{\max }\right\}, \\
& \Lambda_{\mathrm{C} 2}=\left\{\mathbf{q} \in \Lambda_{C} \mid \lambda_{r}(\mathbf{q})=\lambda_{\max }\right\}, \\
& \Lambda_{\mathrm{C} 3}=\left\{\mathbf{q} \in \Lambda_{C} \mid \operatorname{Re} \lambda_{c}(\mathbf{q})=\lambda_{\max }, \lambda_{r}(\mathbf{q})=\lambda_{\max }\right\} .
\end{aligned}
$$

Let $e^{\mathfrak{M} t} \mathbf{w}_{0}(\mathbf{x})$ be the dominant part of the solution $e^{\mathfrak{L} t} \mathbf{w}_{0}(\mathbf{x})$ of the linearied system (5) and

$$
\begin{aligned}
e^{\mathfrak{M} t} \mathbf{w}_{0}(\mathbf{x})= & \sum_{\mathbf{q} \in \Lambda_{R 1}} \sum_{i \in I_{1}} w_{i}(\mathbf{q}) \mathbf{r}_{i}(\mathbf{q}) e^{\lambda_{\max }} e_{\mathbf{q}}(\mathbf{x}) \\
& +\sum_{\mathbf{q} \in \Lambda_{\mathrm{C} 1}}\left[w^{\operatorname{Re}}(\mathbf{q})\left(\operatorname{Rer}_{c}(\mathbf{q}) \cos \left[\left(\operatorname{Im} \lambda_{c}(\mathbf{q})\right) t\right]-\operatorname{Imr}_{c}(\mathbf{q}) \sin \left[\left(\operatorname{Im} \lambda_{c}(\mathbf{q})\right) t\right]\right)\right. \\
& \left.+w^{\operatorname{Im}}(\mathbf{q})\left(\operatorname{Rer}_{c}(\mathbf{q}) \sin \left[\left(\operatorname{Im} \lambda_{c}(\mathbf{q})\right) t\right]+\operatorname{Imr}_{c}(\mathbf{q}) \cos \left[\left(\operatorname{Im} \lambda_{c}(\mathbf{q})\right) t\right]\right)\right] e^{\lambda_{\max } t} \\
& +\sum_{\mathbf{q} \in \Lambda_{C 2}} w_{r}(\mathbf{q}) \mathbf{r}_{r}(\mathbf{q}) e^{\lambda_{\max } t} e_{\mathbf{q}}(\mathbf{x})
\end{aligned}
$$




$$
\begin{aligned}
& +\sum_{\mathbf{q} \in \Lambda_{C 3}}\left\{\left[w^{\operatorname{Re}}(\mathbf{q})\left(\operatorname{Rer}_{c}(\mathbf{q}) \cos \left[\left(\operatorname{Im} \lambda_{c}(\mathbf{q})\right) t\right]-\operatorname{Im} \mathbf{r}_{c}(\mathbf{q}) \sin \left[\left(\operatorname{Im} \lambda_{c}(\mathbf{q})\right) t\right]\right)\right.\right. \\
& \left.+w^{\operatorname{Im}}(\mathbf{q})\left(\operatorname{Rer}_{c}(\mathbf{q}) \sin \left[\left(\operatorname{Im} \lambda_{c}(\mathbf{q})\right) t\right]+\operatorname{Im} \mathbf{r}_{c}(\mathbf{q}) \cos \left[\left(\operatorname{Im} \lambda_{c}(\mathbf{q})\right) t\right]\right)\right] \\
& \left.+w_{r}(\mathbf{q}) \mathbf{r}_{r}(\mathbf{q})\right\} e^{\lambda_{\max } t} e_{\mathbf{q}}(\mathbf{x}) .
\end{aligned}
$$

Since $\lambda_{1}(\mathbf{q}), \lambda_{2}(\mathbf{q}), \lambda_{3}(\mathbf{q})$ are the roots of $(7)$, let $\beta_{i}(\mathbf{q})=\frac{1}{q^{2}} \lambda_{i}(\mathbf{q})$, then $\beta_{1}(\mathbf{q}), \beta_{2}(\mathbf{q}), \beta_{3}(\mathbf{q})$ are the three roots of $\mathbf{F}_{q}\left(\beta_{\mathbf{q}}\right)=\operatorname{det}\left(\beta_{\mathbf{q}} \mathrm{I}-\frac{1}{q^{2}} \mathbf{L}_{q}\right)=0$ and

$$
\begin{aligned}
\mathbf{F}_{q}\left(\beta_{\mathbf{q}}\right) & =\operatorname{det}\left(\begin{array}{ccc}
\beta_{\mathbf{q}}+d_{1}+\frac{\mu}{q^{2}} & -\chi & \xi \\
-\alpha & \beta_{\mathbf{q}}+d_{2}+\frac{\beta}{q^{2}} & 0 \\
-\gamma & 0 & \beta_{\mathbf{q}}+d_{3}+\frac{\eta}{q^{2}}
\end{array}\right) \\
& =\beta_{\mathbf{q}}^{3}+\bar{b}_{2}(\mathbf{q}) \beta_{\mathbf{q}}^{2}+\bar{b}_{1}(\mathbf{q}) \beta_{\mathbf{q}}+\bar{b}_{0}(\mathbf{q})
\end{aligned}
$$

with

$$
\left\{\begin{array}{l}
\bar{b}_{2}(\mathbf{q})=\left(d_{1}+d_{2}+d_{3}\right)+\frac{1}{q^{2}}(\mu+\beta+\eta), \\
\bar{b}_{1}(\mathbf{q})=\left(d_{1} d_{2}-\alpha \chi-\gamma \xi+d_{1} d_{3}+d_{2} d_{3}+\alpha \chi+\gamma \xi\right), \\
+\frac{1}{q^{2}}\left[\mu\left(d_{2}+d_{3}\right) \quad+\beta\left(d_{1}+d_{3}\right)+\eta\left(d_{1}+d_{2}\right)\right]+\frac{1}{q^{4}}(\mu \beta+\beta \eta+\mu \eta), \\
\bar{b}_{0}(\mathbf{q})=d_{1} d_{2} d_{3}-\alpha \chi d_{3}-\gamma \xi d_{2}+\frac{1}{q^{2}}\left(\mu d_{2} d_{3}+\beta d_{1} d_{2}+\eta d_{1} d_{2}\right)+\frac{1}{q^{4}}\left[\mu \eta d_{2}+\beta \eta d_{2}+\mu \beta d_{3}\right]+\frac{\mu \beta \eta}{q^{6}} .
\end{array}\right.
$$

Moreover,

$$
\left\{\begin{array}{l}
\lim _{q^{2} \rightarrow \infty} \bar{b}_{2}(\mathbf{q})=d_{1}+d_{2}+d_{3}:=\bar{b}_{2}, \\
\lim _{q^{2} \rightarrow \infty} \bar{b}_{1}(\mathbf{q})=d_{1} d_{2}+d_{1} d_{3}+d_{2} d_{3}:=\bar{b}_{1}, \\
\lim _{q^{2} \rightarrow \infty} \bar{b}_{0}(\mathbf{q})=d_{1} d_{2} d_{3}:=\bar{b}_{0} .
\end{array}\right.
$$

One can define a function $\mathbf{F}^{*}\left(\beta_{\mathbf{q}}\right)$ of the form

$$
\mathbf{F}^{*}\left(\beta_{\mathbf{q}}\right):=\beta_{\mathbf{q}}^{3}+\bar{b}_{2} \beta_{\mathbf{q}}^{2}+\bar{b}_{1} \beta_{\mathbf{q}}+\bar{b}_{0}=\left(\beta_{\mathbf{q}}+d_{1}\right)\left(\beta_{\mathbf{q}}+d_{2}\right)\left(\beta_{\mathbf{q}}+d_{3}\right) .
$$

It is clear from the assumption $\left(\mathbf{H}_{2}\right)$ that the equation $\mathbf{F}^{*}\left(\beta_{\mathbf{q}}\right)=0$ has different negative roots $-d_{1},-d_{2},-d_{3}$. For $q^{2}$ sufficiently large, it follows from Lemma 3 that $\operatorname{Re} \beta_{i}(\mathbf{q})<0, \forall 1 \leq i \leq 3$. Thus

$$
0>\operatorname{Re} \beta_{i}(\mathbf{q})>\sum_{j=1}^{3} \operatorname{Re} \beta_{j}(\mathbf{q})=-\operatorname{Re} \bar{b}_{2}(\mathbf{q})
$$

and

$$
\bar{b}_{1}(\mathbf{q})=\beta_{1}(\mathbf{q}) \beta_{2}(\mathbf{q})+\beta_{1}(\mathbf{q}) \beta_{3}(\mathbf{q})+\beta_{2}(\mathbf{q}) \beta_{3}(\mathbf{q}) \geq\left(\operatorname{Im} \beta_{i}(\mathbf{q})\right)^{2} .
$$

For $q^{2}$ large enough, by (18) and (19), we have

$$
0>\operatorname{Re} \beta_{i}(\mathbf{q})>-\bar{b}_{2}-1>-\infty .
$$

Again combining (18) and (20) yields for $q^{2}$ sufficiently large

$$
\left|\operatorname{Im} \beta_{i}(\mathbf{q})\right|<\sqrt{\bar{b}_{1}+1}<+\infty .
$$

Applying (21) and (22), for every sequence $\left\{\mathbf{q}_{m}\right\} \in \mathbb{N}^{d}$, there exists a subsequence of $\left\{\mathbf{q}_{n}\right\}$ such that for $1 \leq i \leq 3$ there exist limits

$$
\lim _{n \rightarrow \infty} \operatorname{Re} \beta_{i}\left(\mathbf{q}_{n}\right), \lim _{n \rightarrow \infty} \operatorname{Im} \beta_{i}\left(\mathbf{q}_{n}\right) .
$$


Hence

$$
\lim _{n \rightarrow \infty} \beta_{i}\left(\mathbf{q}_{n}\right)=\beta_{i} \in \mathbb{C} .
$$

Notice by (18) and (23) that

$$
\left\{\begin{array}{l}
-\left(\beta_{1}+\beta_{2}+\beta_{3}\right)=\bar{b}_{2}=d_{1}+d_{2}+d_{3} \\
\beta_{1} \beta_{2}+\beta_{1} \beta_{3}+\beta_{2} \beta_{3}=\bar{b}_{1}=d_{1} d_{2}+d_{1} d_{3}+d_{2} d_{3} \\
-\beta_{1} \beta_{2} \beta_{3}=\bar{b}_{0}=d_{1} d_{2} d_{3} .
\end{array}\right.
$$

This means that $\left\{\beta_{1}, \beta_{2}, \beta_{3}\right\}$ is a permutation of $\left\{-d_{1},-d_{2},-d_{3}\right\}$. So for every sequence $\left\{\mathbf{q}_{n}\right\} \in \mathbb{N}^{d}$, there exists a subsequence $\left\{\mathbf{q}_{n_{j}}\right\}$ such that

$$
\lim _{j \rightarrow \infty} \beta_{i}\left(\mathbf{q}_{n_{j}}\right)=\beta_{i} .
$$

Hence we can assume that

$$
\lim _{q^{2} \rightarrow \infty} \beta_{i}(\mathbf{q})=-d_{i}, \forall 1 \leq i \leq 3,
$$

or equivalently

$$
\lim _{q^{2} \rightarrow \infty} \frac{1}{q^{2}} \lambda_{i}(\mathbf{q})=-d_{i}, \forall 1 \leq i \leq 3 .
$$

Using the similar arguments of Lemma 4 in Hoang [? ], the following lemma can be derived.

Lemma 4. If $\mathbf{q} \in \mathbb{N}^{d}$ and $q^{2}$ sufficiently large, then $\lambda_{1}(\mathbf{q}), \lambda_{2}(\mathbf{q}), \lambda_{3}(\mathbf{q})$ are real numbers and $\lambda_{i}(\mathbf{q}) \neq \lambda_{j}(\mathbf{q})$, $i \neq j, i, j=1,2,3$

Proof. It follows from the assumptions $\left(\mathbf{H}_{2}\right)$ and (25) that $\operatorname{Re} \lambda_{i}(\mathbf{q}) \neq \operatorname{Re} \lambda_{j}(\mathbf{q}), i \neq j$. If there exists a sequence $\left\{\mathbf{q}_{n}\right\} \in \mathbb{N}^{d}$ such that the sequence $\lambda_{i_{n}}\left(\mathbf{q}_{n}\right) \notin \mathbb{R}$, then we can choose a subsequence $\left\{n_{m}\right\}$ of $\{n\}$ and an integer $j, 1 \leq j \leq 3$ such that $i_{n_{m}} \equiv j$. Hence

$$
\lim _{q_{n_{m}}^{2} \rightarrow \infty} \frac{1}{q_{n_{m}}^{2}} \lambda_{j}\left(\mathbf{q}_{n_{m}}\right)=-d_{j}
$$

and

$$
\lim _{q_{n_{m}}^{2} \rightarrow \infty} \frac{1}{q_{n_{m}}^{2}} \overline{\lambda_{j}\left(\mathbf{q}_{n_{m}}\right)}=-d_{j}
$$

where $\overline{\lambda_{j}\left(\mathbf{q}_{n_{m}}\right)}$ is the complex conjugation of $\lambda_{j}\left(\mathbf{q}_{n_{m}}\right)$.

Notice that $\overline{\lambda_{1}\left(\mathbf{q}_{n_{m}}\right)} \in\left\{\lambda_{2}\left(\mathbf{q}_{n_{m}}\right), \lambda_{3}\left(\mathbf{q}_{n_{m}}\right)\right\}, \overline{\lambda_{2}\left(\mathbf{q}_{n_{m}}\right)} \in\left\{\lambda_{1}\left(\mathbf{q}_{n_{m}}\right), \lambda_{3}\left(\mathbf{q}_{n_{m}}\right)\right\}$ and $\overline{\lambda_{3}\left(\mathbf{q}_{n_{m}}\right)} \in$ $\left\{\lambda_{1}\left(\mathbf{q}_{n_{m}}\right), \lambda_{2}\left(\mathbf{q}_{n_{m}}\right)\right\}$, then there exists a subsequence of $\left\{n_{m}\right\}$, still denoted by $\left\{n_{m}\right\}$ and $1 \leq l \leq 3, l \neq j$ such that $\overline{\lambda_{j}\left(\mathbf{q}_{n_{m}}\right)}=\lambda_{l}\left(\mathbf{q}_{n_{m}}\right)$, one can obtain

$$
-d_{j}=\lim _{q_{n_{m}}^{2} \rightarrow \infty} \frac{1}{q_{n_{m}}^{2}} \overline{\lambda_{j}\left(\mathbf{q}_{n_{m}}\right)}=\lim _{q_{n_{m}}^{2} \rightarrow \infty} \frac{1}{q_{n_{m}}^{2}} \lambda_{l}\left(\mathbf{q}_{n_{m}}\right)=-d_{l}, \forall m \in \mathbb{N} .
$$

So $d_{j}=d_{l}$ and $j \neq l$, in contradiction to the assumption $\left(\mathbf{H}_{2}\right)$. Therefore, for $q^{2}$ sufficiently large $\lambda_{1}(\mathbf{q})$, $\lambda_{2}(\mathbf{q}), \lambda_{3}(\mathbf{q})$ are real numbers, and we deduce by $\operatorname{Re} \lambda_{i}(\mathbf{q}) \neq \operatorname{Re} \lambda_{j}(\mathbf{q})$ that $\lambda_{i}(\mathbf{q}) \neq \lambda_{j}(\mathbf{q})$ whenever $i \neq j$, which completes the proof.

\section{Growing modes and Bootstrap lemma}

\subsection{Growing modes in the model (1)}

For convenience we will always denote universal positive constants depending on $d_{i}, \chi, \xi, \mu, \alpha, \beta, \gamma, \eta$ $(i=1,2,3)$ by $C_{k}(k=1,2, \cdots)$. Norm in $L^{2}\left(\mathbb{T}^{d}\right)$ is denoted by $\|\cdot\|$. 
Lemma 5. Suppose that $\left(\boldsymbol{H}_{1}\right)$ and $\left(\boldsymbol{H}_{3}\right)$ hold, and $\mathbf{w}(\mathbf{x}, t) \equiv e^{\mathfrak{L} t} \mathbf{w}_{0}(\mathbf{x})$ is a solution to the linearized system (5) with initial condition $\mathbf{w}_{0}(\mathbf{x})$. Then there exists a constant $\hat{C}_{1}>0$ depending on $d_{i}, \chi, \xi, \mu, \alpha, \beta, \gamma, \eta(i=1,2,3)$ such that

$$
\|\mathbf{w}(\cdot, t)\| \leq \hat{C}_{1} e^{\lambda_{\max } t}\|\mathbf{w}(\cdot, 0)\|, \forall t \geq 0 .
$$

Proof. We will proceed in the following two cases.

Case 1: For $\mathbf{t} \geq 0, \mathbf{q} \in \mathbb{N}^{d}, q^{2}$ sufficiently large. By Lemma 4 , for $q^{2}$ sufficiently large, the matrix $\mathbf{L}_{q}$ has three distinct eigenvalues $\lambda_{1}(\mathbf{q}), \lambda_{2}(\mathbf{q}), \lambda_{3}(\mathbf{q})$ and the corresponding linearly independent eigenvectors $\mathbf{r}_{1}(\mathbf{q}), \mathbf{r}_{2}(\mathbf{q})$, $\mathbf{r}_{3}(\mathbf{q})$. We first look for eigenvector $\mathbf{r}_{1}(\mathbf{q})$ such that

$$
\mathbf{r}_{1}(\mathbf{q})=\left(1, r_{12}(\mathbf{q}), r_{13}(\mathbf{q})\right)^{\mathrm{T}},
$$

where $r_{12}(\mathbf{q}), r_{13}(\mathbf{q})$ are the solutions of the linear system

$$
\begin{gathered}
\left(-d_{2} q^{2}-\beta-\lambda_{1}(\mathbf{q})\right) r_{12}(\mathbf{q})+0=-\alpha, \\
0+\left(-d_{3} q^{2}-\eta-\lambda_{1}(\mathbf{q})\right) r_{13}(\mathbf{q})=-\gamma . \\
r_{12}(\mathbf{q})=\frac{\alpha}{\left(d_{2} q^{2}+\beta+\lambda_{1}(\mathbf{q})\right)}, \\
r_{13}(\mathbf{q})=\frac{\gamma}{\left(d_{3} q^{2}+\eta+\lambda_{1}(\mathbf{q})\right)}, \\
\lim _{q^{2} \rightarrow \infty} r_{12}=0, \\
\lim _{q^{2} \rightarrow \infty} r_{13}=0,
\end{gathered}
$$

hence

$$
\lim _{q^{2} \rightarrow \infty} \mathbf{r}_{1}=(1,0,0)^{\mathrm{T}}
$$

Let $\mathbf{r}_{2}(\mathbf{q})=\left(r_{21}(\mathbf{q}), 1, r_{23}(\mathbf{q})\right)^{\mathrm{T}}, \mathbf{r}_{3}(\mathbf{q})=\left(r_{31}(\mathbf{q}), r_{32}(\mathbf{q}), 1\right)^{\mathrm{T}}$ be eigenvectors corresponding to the eigenvalues $\lambda_{2}(\mathbf{q}), \lambda_{3}(\mathbf{q})$, respectively. Then

$$
\lim _{q^{2} \rightarrow \infty} r_{21}(\mathbf{q}) q^{2}=\frac{\chi}{\left(d_{2}-d_{1}\right)}, \lim _{q^{2} \rightarrow \infty} r_{23}(\mathbf{q}) q^{2}=0,
$$

and

$$
\lim _{q^{2} \rightarrow \infty} r_{31}(\mathbf{q}) q^{2}=\frac{-\xi}{\left(d_{3}-d_{1}\right)}, \lim _{q^{2} \rightarrow \infty} r_{32}(\mathbf{q}) q^{2}=0
$$

Therefore

$$
\lim _{q^{2} \rightarrow \infty} \mathbf{r}_{2}(\mathbf{q})=\left(\frac{\chi}{\left(d_{2}-d_{3}\right)}, 1,0\right)^{\mathrm{T}}, \lim _{q^{2} \rightarrow \infty} \mathbf{r}_{3}(\mathbf{q})=\left(\frac{-\xi}{\left(d_{1}-d_{3}\right)}, 0,1\right)^{\mathrm{T}} .
$$

By (27) and (28), we deduce that there exists a constant $C_{1}>0$ such that

$$
\left|\mathbf{r}_{i}(\mathbf{q})\right| \leq C_{1}, \forall \mathbf{q} \in \Omega, i=1,2,3 .
$$

For $q^{2}$ sufficiently large, it is follows from (13) that $\mathbf{w}_{\mathbf{q}}=\sum_{i=1}^{3} w_{i}(\mathbf{q}) \mathbf{r}_{i}(\mathbf{q})$. Based on Cramer's Rule and Hadamard inequality, we have

$$
\left\{\begin{array}{l}
\left|w_{1}(\mathbf{q})\right| \leq \frac{\left|\mathbf{r}_{2}(\mathbf{q})\right| \times\left|\mathbf{r}_{3}(\mathbf{q})\right| \times\left|\mathbf{w}_{\mathbf{q}}\right|}{\left|\operatorname{det}\left[\mathbf{r}_{1}(\mathbf{q}), \mathbf{r}_{2}(\mathbf{q}), \mathbf{r}_{3}(\mathbf{q})\right]\right|} \\
\left|w_{2}(\mathbf{q})\right| \leq \frac{\left|\mathbf{r}_{1}(\mathbf{q})\right| \times\left|\mathbf{r}_{3}(\mathbf{q})\right| \times\left|\mathbf{w}_{\mathbf{q}}\right|}{\left|\operatorname{det}\left[\mathbf{r}_{1}(\mathbf{q}), \mathbf{r}_{2}(\mathbf{q}), \mathbf{r}_{3}(\mathbf{q})\right]\right|} \\
\left|w_{3}(\mathbf{q})\right| \leq \frac{\left|\mathbf{r}_{1}(\mathbf{q})\right| \times\left|\mathbf{r}_{2}(\mathbf{q})\right| \times\left|\mathbf{w}_{\mathbf{q}}\right|}{\left|\operatorname{det}\left[\mathbf{r}_{1}(\mathbf{q}), \mathbf{r}_{2}(\mathbf{q}), \mathbf{r}_{3}(\mathbf{q})\right]\right|}
\end{array}\right.
$$


In terms of (27) and (28), one can obtain

$$
\lim _{q^{2} \rightarrow \infty} \operatorname{det}\left[\mathbf{r}_{1}(\mathbf{q}), \mathbf{r}_{2}(\mathbf{q}), \mathbf{r}_{3}(\mathbf{q})\right]=1 .
$$

Applying (30) and (31) yields

$$
\left|w_{i}(\mathbf{q})\right| \leq C_{2}\left|\mathbf{w}_{\mathbf{q}}\right|, \forall \mathbf{q} \in \Omega, i=1,2,3,
$$

where $C_{2}:=\max \left\{1, \sqrt{\left(\frac{x}{d_{2}-d_{1}}\right)^{2}+1}, \sqrt{\left(\frac{\xi}{d_{2}-d_{3}}\right)^{2}+1}\right\}>0$. Then, using (29), (32) and $\lambda_{i}(\mathbf{q}) \leq \lambda_{\max }$, this shows that for $q^{2}$ sufficiently large there exists a constant $C_{3}>0$ independent of $\mathbf{q}$ such that

$$
\left|w_{i}(\mathbf{q}) \mathbf{r}_{i}(\mathbf{q}) e^{\lambda_{i}(\mathbf{q}) t}\right| \leq C_{1} C_{2} e^{\lambda_{\max } t}\left|\mathbf{w}_{\mathbf{q}}\right|
$$

which leads to

$$
\left\|\sum_{i=1}^{3} w_{i}(\mathbf{q}) \mathbf{r}_{i}(\mathbf{q}) e^{\lambda_{i}(\mathbf{q}) t} e_{\mathbf{q}}(\mathbf{x})\right\|^{2} \leq 9 C_{3}^{2}\left(\frac{\pi}{2}\right)^{d} e^{2 \lambda_{\max } t}\left|\mathbf{w}_{\mathbf{q}}\right|^{2} .
$$

Case 2: For $t \leq 1$. It is sufficiently to derive standard estimate in $\mathbf{L}^{2}$. From Neumann boundary condition, we can multiplying the first equation in (6) by $u_{1}$, the second equation by $k u_{2}$ and the third by $u_{3}$, adding them together, and integrating the result in $\mathbb{T}^{d}$, we have

$$
\begin{aligned}
& \frac{1}{2} \frac{d}{d t} \int_{\mathbb{T}^{d}}\left\{\left|u_{1}\right|^{2}+k\left|u_{2}\right|^{2}+\left|u_{3}\right|^{2}\right\} \mathbf{d} x+\int_{\mathbb{T}^{d}}\left\{d_{1}|\nabla u+1|^{2}+k d_{2}\left|\nabla u_{2}\right|^{2}+d_{3}\left|\nabla u_{3}\right|^{2}-\chi\left(\nabla u_{1} \nabla u_{2}\right)+\xi\left(\nabla u_{1} \nabla u_{3}\right)\right\} \mathbf{d} x \\
& =-\mu \int_{\mathbb{T}^{d}} u_{1}^{2} \mathbf{d} x-k \beta \int_{\mathbb{T}^{d}} u_{2}^{2} \mathbf{d} x-\eta \int_{\mathbb{T}^{d}} u_{3}^{2} \mathbf{d} x+\alpha k \int_{\mathbb{T}^{d}} u_{1} u_{2} \mathbf{d} x+\gamma \int_{\mathbb{T}^{d}} u_{1} u_{3} \mathbf{d} x .
\end{aligned}
$$

where $k=\frac{\chi^{2} d_{3}}{d_{1} d_{2} d_{3}+d_{2} \xi^{2}}$.

Then the integrand of the second integral can be estimated as follows

$$
\begin{aligned}
& d_{1}|\nabla u+1|^{2}+k d_{2}\left|\nabla u_{2}\right|^{2}+d_{3}\left|\nabla u_{3}\right|^{2}-\chi\left(\nabla u_{1} \nabla u_{2}\right)+\xi\left(\nabla u_{1} \nabla u_{3}\right) \\
& \geq \frac{d_{1}}{2}\left|\nabla u_{1}\right|^{2}+\frac{k d_{2}}{2}\left|\nabla u_{2}\right|^{2}+\frac{3 d_{3}}{2}\left|\nabla u_{3}\right|^{2} \geq 0
\end{aligned}
$$

Using Young inequality, we deduce that

$$
\begin{aligned}
& -\mu \int_{\mathbb{T}^{d}} u_{1}^{2} \mathbf{d} x-k \beta \int_{\mathbb{T}^{d}} u_{2}^{2} \mathbf{d} x-\eta \int_{\mathbb{T}^{d}} u_{3}^{2} \mathbf{d} x+\alpha k \int_{\mathbb{T}^{d}} u_{1} u_{2} \mathbf{d} x+\gamma \int_{\mathbb{T}^{d}} u_{1} u_{3} \mathbf{d} x \\
& \leq\left(-\mu+\frac{k \alpha^{2}}{2 \beta}+\frac{v^{2}}{2 \eta}\right)\left|u_{1}\right|^{2}-\frac{k \beta}{2}\left|u_{2}\right|^{2}-\frac{\eta}{2}\left|u_{3}\right|^{2} \\
& \leq \max \left(-\mu+\frac{k \alpha^{2}}{2 \beta}+\frac{\gamma^{2}}{2 \eta},-\frac{\beta}{2}, \frac{\eta}{2}\right) \int_{\mathbb{T}^{d}}\left(\left|u_{1}\right|^{2}+k\left|u_{2}\right|^{2}+\left|u_{3}\right|^{2}\right) \mathbf{d} x .
\end{aligned}
$$

Then

$$
\frac{1}{2} \frac{d}{d t} \int_{\mathbb{T}^{d}}\left\{\left|u_{1}\right|^{2}+k\left|u_{2}\right|^{2}+\left|u_{3}\right|^{2}\right\} \mathbf{d} x \leq \max \left(-\mu+\frac{k \alpha^{2}}{2 \beta}+\frac{\gamma^{2}}{2 \eta},-\frac{\beta}{2}, \frac{\eta}{2}\right) \int_{\mathbb{T}^{d}}\left(\left|u_{1}\right|^{2}+k\left|u_{2}\right|^{2}+\left|u_{3}\right|^{2}\right) \mathbf{d} x .
$$

By Grownwall inequality, we can obtain $\|\mathbf{w}(\cdot, t)\| \leq \hat{C}_{1} e^{\lambda_{\max } t}\|\mathbf{w}(\cdot, 0)\|$, where $\hat{C}_{1}=\max \left(-\mu+\frac{k \alpha^{2}}{2 \beta}+\right.$ $\left.\frac{\gamma^{2}}{2 \eta},-\frac{\beta}{2}, \frac{\eta}{2}\right)$. This completes the proof.

\subsection{Bootstrap lemma and $H^{2}$-estimate in the model (1)}

Denote

$$
\partial_{x_{i} x_{j}} u=\frac{\partial^{2} u}{\partial x_{i} \partial x_{j}}, \partial_{x_{i}} u=\frac{\partial u}{\partial x_{i}}, D^{\alpha} u=\frac{\partial^{|\alpha|} u}{\partial x_{1}^{\alpha_{1}} \cdots \partial x_{d}^{\alpha_{d}}}
$$


where $\alpha=\left(\alpha_{1}, \cdots, \alpha_{d}\right),|\alpha|=\sum_{i=1}^{d} \alpha_{i}, i, j=1, \cdots, d$. Let us introduce

$$
k=\frac{\chi^{2} d_{3}}{d_{1} d_{2} d_{3}+d_{2} \xi^{2}}
$$

By standard theory of parabolic equation, we can establish the existence of local solutions for the model (4).

Lemma 6. (Local existence). For $s \geq 1(d=1)$ and $s \geq 2(d=2,3)$, there exist a $T_{0}>0$ such that the problem (4) with $u_{1}(\cdot, 0), u_{2}(\cdot, 0), u_{3}(\cdot, 0) \in H^{s}\left(\mathbb{T}^{d}\right)$ has a unique solution $\mathbf{w}(\cdot, t)$ on $\left(0, T_{0}\right)$ which satisfies

$$
\|\mathbf{w}(t)\|_{H^{s}\left(\mathbb{T}^{d}\right)} \leq C\|\mathbf{w}(0)\|_{H^{s}\left(\mathbb{T}^{d}\right)},
$$

where $C$ is a positive constant depending on $d_{i}, \xi, \chi, \alpha, \beta, \gamma, \eta(i=1,2,3)$.

Lemma 7. Let $\mathbf{w}(\mathbf{x}, t)=\left(u_{1}(\mathbf{x}, t), u_{2}(\mathbf{x}, t), v(\mathbf{x}, t)\right)^{\mathrm{T}}$ be a solution of the nonlinear perturbation system (3). Then

$$
\begin{aligned}
& \frac{1}{2} \frac{d}{d t} \sum_{|\alpha|=2} \int_{\mathbb{T}^{d}}\left\{\left|D^{\alpha} u_{1}\right|^{2}+k\left|D^{\alpha} u_{2}\right|^{2}+\left|D^{\alpha} u_{3}\right|^{2}\right\} d \mathbf{x} \\
& +\sum_{|\alpha|=2} \int_{\mathbb{T}^{d}}\left\{\frac{d_{1}}{4}\left|\nabla\left(D^{\alpha} u_{1}\right)\right|^{2}+\frac{k d_{2}}{2}\left|\nabla\left(D^{\alpha} u_{2}\right)\right|^{2}+\frac{3 d_{3}}{2}\left|\nabla\left(D^{\alpha} u_{3}\right)\right|^{2}\right\} d \mathbf{x} \\
& +\frac{\beta k}{2} \sum_{|\alpha|=2} \int_{\mathbb{T}^{d}}\left|D^{\alpha} u_{2}\right|^{2} d \mathbf{x}+\frac{\eta}{2} \sum_{|\alpha|=2} \int_{\mathbb{T}^{d}}\left|D^{\alpha} u_{3}\right|^{2} d \mathbf{x} \\
& \leq \hat{C}_{2}\|\mathbf{w}\|_{H^{2}\left(\mathbb{T}^{d}\right)}|| \nabla^{3} \mathbf{w}\left\|^{2}+\hat{C}_{3}\right\| u_{1} \|^{2},
\end{aligned}
$$

where $\hat{C}_{2}$ and $C_{0}$ are the generic constants and $\hat{C}_{3}=\left(\frac{\alpha^{2} \eta k+v^{2}}{8 \beta \eta a^{2}}\right) c_{0}$.

Proof. Let $\mathbf{w}(\mathbf{x}, t)$ be a solution of (4). It is not hard to verify that if $\tilde{\mathbf{w}}(\mathbf{x}, t)=\left(\tilde{u}_{1}(\mathbf{x}, t), \tilde{u}_{2}(\mathbf{x}, t), \tilde{u}_{3}(\mathbf{x}, t)\right)^{\mathrm{T}}$ is the even extension of $\mathbf{w}(\mathbf{x}, t)$ on $2 \mathbb{T}^{d}=(-\pi, \pi)^{d}(d=1,2,3)$. The $\tilde{\mathbf{w}}(\mathbf{x}, t)$ is also the solution of (4) with the homogeneous Neumann boundary conditions and periodical boundary conditions on $2 \mathbb{T}^{d}$.

Therefore,

$$
\begin{aligned}
& \frac{1}{2} \frac{d}{d t} \int_{2 \mathbb{T}^{d}}\left[\left|\partial_{x_{i} x_{j}} \tilde{u}_{1}\right|^{2}+k\left|\partial_{x_{i} x_{j}} \tilde{u}_{2}\right|^{2}+\left|\partial_{x_{i} x_{j}} \tilde{u}_{3}\right|^{2}\right] d \mathbf{x}+\int_{2 \mathbb{T}^{d}}\left[d_{1}\left|\nabla\left(\partial_{x_{i} x_{j}} \tilde{u}_{1}\right)\right|^{2}+k d_{2}\left|\nabla\left(\partial_{x_{i} x_{j}} \tilde{u}_{2}\right)\right|^{2}+d_{3}\left|\nabla\left(\partial_{x_{i} x_{j}} \tilde{u}_{3}\right)\right|^{2}\right. \\
& \left.-\chi \nabla\left(\partial_{x_{i} x_{j}} \tilde{u}_{1}\right) \cdot \nabla\left(\partial_{x_{i} x_{j}} \tilde{u}_{2}\right)+\xi \nabla\left(\partial_{x_{i} x_{j}} \tilde{u}_{1}\right) \cdot \nabla\left(\partial_{x_{i} x_{j}} \tilde{u}_{3}\right)\right] d \mathbf{x} \\
& +\mu \int_{2 \mathbb{T}^{d}}\left|\partial_{x_{i} x_{j}} \tilde{u}_{1}\right|^{2} d \mathbf{x}+k \beta \int_{2 \mathbb{T}^{d}}\left|\partial_{x_{i} x_{j}} \tilde{u}_{2}\right|^{2} d \mathbf{x}+\eta \int_{2 \mathbb{T}^{d}}\left|\partial_{x_{i} x_{j}} \tilde{u}_{3}\right|^{2} d \mathbf{x} \\
& =\int_{2 \mathbb{T}^{d}}\left[\chi \nabla\left(\partial_{x_{i} x_{j}} \tilde{u}_{1}\right) \cdot \partial_{x_{i} x_{j}}\left(\tilde{u}_{1} \nabla \tilde{u}_{2}\right)-\xi \nabla\left(\partial_{x_{i} x_{j}} \tilde{u}_{1}\right) \cdot \partial_{x_{i} x_{j}}\left(\tilde{u}_{1} \nabla \tilde{u}_{3}\right)\right] d \mathbf{x} \\
& +\alpha k \int_{2 \mathbb{T}^{d}} \partial_{x_{i} x_{j}} \tilde{u}_{1} \cdot \partial_{x_{i} x_{j}} \tilde{u}_{2} d \mathbf{x}+\gamma \int_{2 \mathbb{T}^{d}} \partial_{x_{i} x_{j}} \tilde{u}_{1} \cdot \partial_{x_{i} x_{j}} \tilde{u}_{3} d \mathbf{x}-2 \mu \int_{2 \mathbb{T}^{d}}\left[u_{1}\left|\partial_{x_{i} x_{j}} \tilde{u}_{1}\right|^{2}+\left|\partial_{x_{i}} \tilde{u}_{1}\right|\left|\partial_{x_{j}} \tilde{u}_{1}\right|\left|\partial_{x_{i} x_{j}} \tilde{u}_{1}\right|\right] d \mathbf{x} \\
& :=J_{1}+J_{2}+J_{3}+J_{4} .
\end{aligned}
$$

Using Young inequality, we get

$$
\begin{aligned}
& {\left[d_{1}\left|\nabla\left(\partial_{x_{i} x_{j}} \tilde{u}_{1}\right)\right|^{2}+k d_{2}\left|\nabla\left(\partial_{x_{i} x_{j}} \tilde{u}_{2}\right)\right|^{2}+d_{3}\left|\nabla\left(\partial_{x_{i} x_{j}} \tilde{u}_{3}\right)\right|^{2}-\chi \nabla\left(\partial_{x_{i} x_{j}} \tilde{u}_{1}\right) \cdot \nabla\left(\partial_{x_{i} x_{j}} \tilde{u}_{2}\right)+\xi \nabla\left(\partial_{x_{i} x_{j}} \tilde{u}_{1}\right) \cdot \nabla\left(\partial_{x_{i} x_{j}} \tilde{u}_{3}\right)\right]} \\
& \geq \frac{d_{1}}{2}\left|\nabla\left(\partial_{x_{i} x_{j}} \tilde{u}_{1}\right)\right|^{2}+\frac{k d_{2}}{2}\left|\nabla\left(\partial_{x_{i} x_{j}} \tilde{u}_{2}\right)\right|^{2}+\frac{3 d_{3}}{2}\left|\nabla\left(\partial_{x_{i} x_{j}} \tilde{u}_{3}\right)\right|^{2} .
\end{aligned}
$$


The nonlinear term $J_{1}$ is bounded by

$$
\begin{aligned}
J_{1} \leq & \chi \int_{2 \mathbb{T}^{d}}\left|\nabla\left(\partial_{x_{i} x_{j}} \tilde{u}_{1}\right)\right|\left|\partial_{x_{i} x_{j}} \tilde{u}_{1} \cdot \nabla \tilde{u}_{2}\right| d \mathbf{x}+\chi \int_{2 \mathbb{T}^{d}}\left|\nabla\left(\partial_{x_{i} x_{j}} \tilde{u}_{1}\right) \| \partial_{x_{j}} \tilde{u}_{1} \cdot \nabla\left(\partial_{x_{i}} \tilde{u}_{2}\right)\right| d \mathbf{x} \\
& +\chi \int_{2 \mathbb{T}^{d}}\left|\nabla\left(\partial_{x_{i} x_{j}} \tilde{u}_{1}\right) \| \partial_{x_{i}} \tilde{u}_{1} \cdot \nabla\left(\partial_{x_{j}} \tilde{u}_{2}\right)\right| d \mathbf{x}+\chi \int_{2 \mathbb{T}^{d}}\left|\nabla\left(\partial_{x_{i} x_{j}} \tilde{u}_{1}\right)\right|\left|\tilde{u}_{1} \nabla\left(\partial_{x_{i} x_{j}} \tilde{u}_{2}\right)\right| d \mathbf{x} \\
& -\xi \int_{2 \mathbb{T}^{d}}\left|\nabla\left(\partial_{x_{i} x_{j}} \tilde{u}_{1}\right)\left\|\partial_{x_{i} x_{j}} \tilde{u}_{1} \cdot \nabla \tilde{u}_{3}\left|d \mathbf{x}-\xi \int_{2 \mathbb{T}^{d}}\right| \nabla\left(\partial_{x_{i} x_{j}} \tilde{u}_{1}\right)\right\| \partial_{x_{j}} \tilde{u}_{1} \cdot \nabla\left(\partial_{x_{i}} \tilde{u}_{3}\right)\right| d \mathbf{x} \\
& -\xi \int_{2 \mathbb{T}^{d}}\left|\nabla\left(\partial_{x_{i} x_{j}} \tilde{u}_{1}\right)\left\|\partial_{x_{i}} \tilde{u}_{1} \cdot \nabla\left(\partial_{x_{j}} \tilde{u}_{3}\right)\left|d \mathbf{x}-\xi \int_{2 \mathbb{T}^{d}}\right| \nabla\left(\partial_{x_{i} x_{j}} \tilde{u}_{1}\right)\right\| \tilde{u}_{1} \nabla\left(\partial_{x_{i} x_{j}} \tilde{u}_{3}\right)\right| d \mathbf{x} \\
\leq & \chi\left\|\nabla \tilde{u}_{2}\right\|_{L^{\infty}\left(2 \mathbb{T}^{d}\right)}\left\|\nabla\left(\partial_{x_{i} x_{j}} \tilde{u}_{1}\right)\right\| \cdot\left\|\partial_{x_{i} x_{j}} \tilde{u}_{1}\right\|-\xi\left\|\nabla \tilde{u}_{3}\right\|_{L^{\infty}\left(2 \mathbb{T}^{d}\right)}\left\|\nabla\left(\partial_{x_{i} x_{j}} \tilde{u}_{1}\right)\right\| \cdot\left\|\partial_{x_{i} x_{j}} \tilde{u}_{1}\right\| \\
& +\chi\left\|\tilde{u}_{1}\right\|_{L^{\infty}\left(2 \mathbb{T}^{d}\right)}\left\|\nabla\left(\partial_{x_{i} x_{j}} \tilde{u}_{1}\right)\right\|\left\|\nabla\left(\partial_{x_{i} x_{j}} \tilde{u}_{2}\right)\right\|-\xi\left\|\tilde{u}_{1}\right\|_{L^{\infty}\left(2 \mathbb{T}^{d}\right)}\left\|\nabla\left(\partial_{x_{i} x_{j}} \tilde{u}_{1}\right)\right\|\left\|\nabla\left(\partial_{x_{i} x_{j}} \tilde{u}_{3}\right)\right\| \\
& +2 \chi \sum_{i=1}^{d}\left\|\nabla \tilde{u}_{1}\right\|_{L^{\infty}\left(2 \mathbb{T}^{d}\right)}\left\|\partial_{x_{i} x_{j}} \tilde{u}_{2}\right\|\left\|\nabla\left(\partial_{x_{i} x_{j}} \tilde{u}_{1}\right)\right\|-2 \xi \sum_{i=1}^{d}\left\|\nabla \tilde{u}_{1}\right\|_{L^{\infty}\left(2 \mathbb{T}^{d}\right)}\left\|\partial_{x_{i} x_{j}} \tilde{u}_{3}\right\|\left\|\nabla\left(\partial_{x_{i} x_{j}} \tilde{u}_{1}\right)\right\| .
\end{aligned}
$$

Recalling that the Sobolev imbedding $H^{2}\left(\mathbb{T}^{d}\right) \hookrightarrow L^{\infty}\left(\mathbb{T}^{d}\right)$ for $d \leq 3$, we have

$$
\begin{aligned}
\|g\|_{L^{\infty}\left(2 \mathbb{T}^{d}\right)} & \leq C_{4}\|g\|_{H^{2}\left(2 \mathbb{T}^{d}\right)^{\prime}}, \\
\|g\|_{L^{4}\left(2 \mathbb{T}^{d}\right)} & \leq C_{5}\|g\|_{H^{2}\left(2 \mathbb{T}^{d}\right)^{\prime}} \\
\|g\|_{L^{6}\left(2 \mathbb{T}^{d}\right)} & \leq C_{6}\|g\|_{H^{2}\left(2 \mathbb{T}^{d}\right)^{\prime}} .
\end{aligned}
$$

Notice that

$$
\left\{\begin{array}{l}
\int_{2 \mathbb{T}^{d}} \nabla \tilde{u}_{1} d \mathbf{x}=\int_{2 \mathbb{T}^{d}} \nabla \tilde{u}_{2} d \mathbf{x}=\int_{2 \mathbb{T}^{d}} \nabla \tilde{u}_{3} d \mathbf{x}=0 \\
\int_{2 \mathbb{T}^{d}} \partial_{x_{i} x_{j}} \tilde{u}_{1} d \mathbf{x}=\int_{2 \mathbb{T}^{d}} \partial_{x_{i} x_{j}} \tilde{u}_{2} d \mathbf{x}=\int_{2 \mathbb{T}^{d}} \partial_{x_{i} x_{j}} \tilde{u}_{3} d \mathbf{x}=0
\end{array}\right.
$$

Moreover, if $g \in H^{1}\left(2 \mathbb{T}^{d}\right)$ with $\int_{2 \mathbb{T}^{d}} g=0$, then

$$
\|g\| \leq(2 \pi)^{\frac{d}{4}}\|g\|_{L^{4}\left(2 \mathbb{T}^{d}\right)} \leq C_{7}\|g\|_{H^{1}\left(2 \mathbb{T}^{d}\right)} \leq C_{8}\|\nabla g\|, d \leq 3 .
$$

It follows from (43) and (44) that

$$
\left\|\partial_{x_{i}} g\right\| \leq C_{9}\left\|\nabla\left(\partial_{x_{i}} g\right)\right\|,\left\|\partial_{x_{i} x_{j}} g\right\| \leq C_{9}\left\|\nabla\left(\partial_{x_{i} x_{j}} g\right)\right\|
$$

and

$$
\left.\|\nabla g\| \leq C_{9}\left(\sum_{i, j=1,2}^{d}\left\|\partial_{x_{i} x_{j}} g\right\|^{2}\right)^{\frac{1}{2}} \leq C_{9}^{2}\left(\sum_{|\alpha|=2} \| \nabla\left(D^{\alpha} g\right)\right) \|^{2}\right)^{\frac{1}{2}} .
$$

Together with (40) and (45), we further get

$$
\|\nabla g\|_{L^{\infty}\left(2 \mathbb{T}^{d}\right)} \leq C_{10}\|\nabla g\|_{H^{2}\left(2 \mathbb{T}^{d}\right)} \leq C_{11}\left\|\nabla^{3} g\right\|_{L^{2}\left(2 \mathbb{T}^{d}\right)} .
$$

Then as a consequence of(40) and (45), one can obtain

$$
\sum_{|\alpha|=2} J_{1} \leq(\chi-\xi) C_{12}\|\tilde{\mathbf{w}}\|_{H^{2}\left(2 \mathbb{T}^{d}\right)}\left\|\nabla^{3} \tilde{\mathbf{w}}\right\|^{2},
$$

where $C_{12}:=C_{4}+(1+2 d) C_{9}$.

Applying interpolation, we can deduce that for all $\varepsilon>0$,

$$
\left\|\partial_{x_{i} x_{j}} \tilde{u}\right\|^{2} \leq C_{0}\left(\varepsilon\left\|\nabla\left(\partial_{x_{i} x_{j}} \tilde{u}\right)\right\|^{2}+\frac{\|\tilde{u}\|^{2}}{4 \varepsilon^{2}}\right) .
$$


By the choice of $\varepsilon>0$ in (48) such that $\left(\frac{\alpha^{2} k \eta+\beta v^{2}}{2 \beta \eta}\right) C_{0} \varepsilon=d_{1} / 4$, then

$$
\begin{aligned}
J_{2}+J_{3} & \leq \alpha k \int_{2 \mathbb{T}^{d}} \partial_{x_{i} x_{j}} \tilde{u}_{1} \cdot \partial_{x_{i} x_{j}} \tilde{u}_{2} d \mathbf{x}+\gamma \int_{2 \mathbb{T}^{d}} \partial_{x_{i} x_{j}} \tilde{u}_{1} \cdot \partial_{x_{i} x_{j}} \tilde{u}_{3} d \mathbf{x} \\
& \leq \frac{\alpha^{2} k \eta+\beta \gamma^{2}}{2 \beta \eta} \int_{2 \mathbb{T}^{d}}\left|\partial_{x_{i} x_{j}} \tilde{u}_{1}\right|^{2} d \mathbf{x}+\frac{\beta k}{2} \int_{2 \mathbb{T}^{d}}\left|\partial_{x_{i} x_{j}} \tilde{u}_{2}\right|^{2} d \mathbf{x}+\frac{\eta}{2} \int_{2 \mathbb{T}^{d}}\left|\partial_{x_{i} x_{j}} \tilde{u}_{3}\right|^{2} d \mathbf{x} \\
& \leq \frac{d_{1}}{4}\left\|\nabla\left(\partial_{x_{i} x_{j}} \tilde{u}_{1}\right)\right\|^{2}+\frac{\beta k}{2} \int_{2 \mathbb{T}^{d}}\left|\partial_{x_{i} x_{j}} \tilde{u}_{2}\right|^{2} d \mathbf{x}+\frac{\eta}{2} \int_{2 \mathbb{T}^{d}}\left|\partial_{x_{i} x_{j}} \tilde{u}_{3}\right|^{2} d \mathbf{x}+\left(\frac{\alpha^{2} k \eta+v^{2} \beta}{8 \beta \eta \varepsilon^{2}}\right) C_{0}\left\|\tilde{u}_{1}\right\|^{2} .
\end{aligned}
$$

Then as a consequence of(40),(41),(42)and (45), one can obtain

$$
\sum_{|\alpha|=2} J_{4} \leq 4 \mu C_{10}\|\widetilde{\mathbf{w}}\|_{H^{2}\left(2 \mathbb{T}^{d}\right)}\left\|\nabla^{3} \widetilde{\mathbf{w}}\right\|^{2} .
$$

Substituting (47), (49)-(50) into (37), we have

$$
\begin{aligned}
& \frac{1}{2} \frac{d}{d t} \sum_{|\alpha|=2} \int_{\mathbb{T}^{d}}\left\{\left|D^{\alpha} u_{1}\right|^{2}+k\left|D^{\alpha} u_{2}\right|^{2}+\left|D^{\alpha} u_{3}\right|^{2}\right\} d \mathbf{x} \\
& +\sum_{|\alpha|=2} \int_{\mathbb{T}^{d}}\left\{\frac{d_{1}}{4}\left|\nabla\left(D^{\alpha} u_{1}\right)\right|^{2}+\frac{k d_{2}}{2}\left|\nabla\left(D^{\alpha} u_{2}\right)\right|^{2}+\frac{3 d_{3}}{2}\left|\nabla\left(D^{\alpha} u_{3}\right)\right|^{2}\right\} d \mathbf{x} \\
& +\frac{\beta k}{2} \sum_{|\alpha|=2} \int_{\mathbb{T}^{d}}\left|D^{\alpha} u_{2}\right|^{2} d \mathbf{x}+\frac{\eta}{2} \sum_{|\alpha|=2} \int_{\mathbb{T}^{d}}\left|D^{\alpha} u_{3}\right|^{2} d \mathbf{x} \\
& \leq \hat{C}_{2}\|\mathbf{w}\|_{H^{2}\left(\mathbb{T}^{d}\right)}\left\|\nabla^{3} \mathbf{w}\right\|^{2}+\hat{C}_{3}\left\|u_{1}\right\|^{2},
\end{aligned}
$$

where $\hat{C}_{2}$ and $C_{0}$ are the generic constants and $\hat{C}_{3}=\left(\frac{\alpha^{2} \eta k+\gamma^{2}}{8 \beta \eta a^{2}}\right) c_{0}$. This completes the proof of Lemma 7 .

Lemma 8. Let $\boldsymbol{w}(\boldsymbol{x}, t)$ be a solution to the system (4) such that for $0 \leq t \leq T$,

$$
\|\mathbf{w}(\cdot, t)\|_{H^{2}\left(\mathbb{T}^{d}\right)} \leq \frac{1}{\hat{C}_{2}} \min \left\{\frac{d_{1}}{4}, \frac{k d_{2}}{2}, \frac{3 d_{3}}{2}\right\}
$$

and

$$
\|\mathbf{w}(\cdot, t)\| \leq 2 \hat{C}_{1} e^{\lambda_{\max }}\|\mathbf{w}(\cdot, 0)\|
$$

Then for $0 \leq t \leq T$,

$$
\|\mathbf{w}(\cdot, t)\|_{H^{2}\left(\mathbb{T}^{d}\right)}^{2} \leq \hat{C}_{4}\left\{\|\mathbf{w}(\cdot, 0)\|_{H^{2}\left(\mathbb{T}^{d}\right)}^{2}+e^{2 \lambda_{\max } t}\|\mathbf{w}(\cdot, 0)\|^{2}\right\},
$$

where $\hat{C}_{4}=\max \left\{\left(1+C_{9}^{2}\right) k, 4 \hat{C}_{1}^{2}\left[1+\hat{C}_{3}\left(1+C_{9}^{2}\right) /\left(2 \lambda_{\max }\right)\right]\right\} \geq 1$, if $k \geq 1 . \hat{C}_{4}=\max \left\{\left(1+C_{9}^{2}\right) / k, 4 \hat{C}_{1}^{2}[1+\right.$ $\left.\left.\hat{C}_{3}\left(1+C_{9}^{2}\right) /\left(2 \lambda_{\max } k\right)\right]\right\} \geq 1$, if $k<1$.

Proof. It follows from (45) that

$$
\|\nabla \mathbf{w}(\cdot, t)\|^{2} \leq C_{9}^{2} \sum_{|\alpha|=2}\left\|D^{\alpha} \mathbf{w}(\cdot, t)\right\|^{2}
$$

So

$$
\|\mathbf{w}(\cdot, t)\|_{H^{2}\left(\mathbb{T}^{d}\right)}^{2} \leq\|\mathbf{w}(\cdot, t)\|^{2}+\left(1+C_{9}^{2}\right) \sum_{|\alpha|=2}\left\|D^{\alpha} \mathbf{w}(\cdot, t)\right\|^{2} .
$$

By Lemma 7 and (51), we infer

$$
\frac{d}{d t} \sum_{|\alpha|=2} \int_{\mathbb{T}^{d}}\left\{\left|D^{\alpha} u_{1}\right|^{2}+k\left|D^{\alpha} u_{2}\right|^{2}+\left|D^{\alpha} u_{3}\right|^{2}\right\} d \mathbf{x} \leq \hat{C}_{3}\left\|u_{1}\right\|^{2}+\leq \hat{C}_{3}\|\mathbf{w}(\cdot, t)\|^{2} .
$$


Integrating (57) and using (52), we conclude

$$
\begin{aligned}
& \frac{1}{2} \sum_{|\alpha|=2} \int_{\mathbb{T}^{d}}\left\{\left|D^{\alpha} u_{1}(\cdot, t)\right|^{2}+k\left|D^{\alpha} u_{2}(\cdot, t)\right|^{2}+\left|D^{\alpha} u_{3}(\cdot, t)\right|^{2}\right\} d \mathbf{x} \\
& \leq \sum_{|\alpha|=2} \int_{\mathbb{T}^{d}}\left\{\left|D^{\alpha} u_{1}(\cdot, 0)\right|^{2}+k\left|D^{\alpha} u_{2}(\cdot, 0)\right|^{2}+\left|D^{\alpha} u_{3}(\cdot, 0)\right|^{2}\right\} d \mathbf{x}+\frac{4 \hat{C}_{1}^{2} \hat{C}_{3}}{\lambda_{\max }} e^{2 \lambda_{\max } t}\|\mathbf{w}(\cdot, 0)\|^{2} .
\end{aligned}
$$

We first consider the case $k \geq 1$. By (57), we have

$$
\sum_{|\alpha|=2}\left\|D^{\alpha} \mathbf{w}(\cdot, t)\right\|^{2} \leq k \sum_{|\alpha|=2}\left\|D^{\alpha} \mathbf{w}(\cdot, 0)\right\|^{2}+\frac{4 \hat{C}_{1}^{2} \hat{C}_{3}}{\lambda_{\max }} e^{2 \lambda_{\max } t}\|\mathbf{w}(\cdot, 0)\|^{2} .
$$

We see from this estimate and (55) that

$$
\|\mathbf{w}(\cdot, t)\|_{H^{2}\left(\mathbb{T}^{d}\right)}^{2} \leq \hat{C}_{4}\left\{\|\mathbf{w}(\cdot, 0)\|_{H^{2}\left(\mathbb{T}^{d}\right)}^{2}+e^{2 \lambda_{\max } t}\|\mathbf{w}(\cdot, 0)\|^{2}\right\},
$$

where $\hat{C}_{4}:=\max \left\{\left(1+C_{9}^{2}\right) k, 4 \hat{C}_{1}^{2}\left[1+\frac{\hat{C}_{3}\left(1+C_{9}^{2}\right)}{\lambda_{\max }}\right]\right\}$.

On the other hand, for $K<1$, we deduce by (57) that

$$
\sum_{|\alpha|=2}\left\|D^{\alpha} \mathbf{w}(\cdot, t)\right\|^{2} \leq \frac{1}{K}\left(\sum_{|\alpha|=2}\left\|D^{\alpha} \mathbf{w}(\cdot, 0)\right\|^{2}+\frac{4 \hat{C}_{1}^{2} \hat{C}_{3}}{\lambda_{\max }} e^{2 \lambda_{\max } t}\|\mathbf{w}(\cdot, 0)\|^{2}\right) .
$$

This estimate, combined with (52) and (55) gives

$$
\|\mathbf{w}(\cdot, t)\|_{H^{2}\left(\mathbb{T}^{d}\right)}^{2} \leq \hat{C}_{4}\left\{\|\mathbf{w}(\cdot, 0)\|_{H^{2}\left(\mathbb{T}^{d}\right)}^{2}+e^{2 \lambda_{\max } t}\|\mathbf{w}(\cdot, 0)\|^{2}\right\},
$$

where $\hat{C}_{4}:=\max \left\{\frac{1+C_{9}^{2}}{k}, 4 \hat{C}_{1}^{2}\left[1+\frac{\hat{C}_{3}\left(1+C_{9}^{2}\right)}{\lambda_{\max } k}\right]\right\}$. This completes the proof of Lemma 8 .

\section{Main result}

Assume $\theta$ be a small fixed constant. For $\delta>0$ arbitrary small, we define the escape time $T^{\delta}$ by

$$
\theta=\delta e^{\lambda_{\max } T^{\delta}}
$$

where $\lambda_{\max }$ is the dominant eigenvalue which is the maximal growth rate (see (10)). Obviously,

$$
T^{\delta}=\frac{1}{\lambda_{\max }} \ln \frac{\theta}{\delta} .
$$

Our main result in this paper is as follows:

Theorem 2. Suppose that $\left(\mathbf{H}_{1}\right),\left(\mathbf{H}_{2}\right)$ and $\left(\mathbf{H}_{3}\right)$ are satisfied. Let $\mathbf{w}_{0}(\mathbf{x}) \in H^{2}\left(\mathbb{T}^{d}\right)$ with $\left\|\mathbf{w}_{0}(\mathbf{x})\right\|=1$. Then there exist constants $\delta_{0}>0, \hat{C}>0$, and $\theta>0$ depending on $d_{i}, \chi, \xi, \mu, \alpha, \beta, \eta, \gamma,(i=1,2,3)$ such that $\forall 0<\delta \leq \delta_{0}$, if the initial perturbation of the steady state $\mathbf{w}_{c}$ is $\mathbf{w}^{\delta}(\cdot, 0)=\delta \mathbf{w}_{0}$, then its nonlinear evolution $\mathbf{w}^{\delta}(\cdot, t)$ satisfies

$$
\left\|\mathbf{w}^{\delta}(\cdot, t)-\delta e^{\mathfrak{M} t} \mathbf{w}_{0}(\mathbf{x})\right\| \leq \hat{C}\left\{e^{-\rho t}+\delta\left\|\mathbf{w}_{0}\right\|_{H^{2}\left(\mathbb{T}^{d}\right)}^{2}+\delta e^{\lambda_{\max } t}\right\} \delta e^{\lambda_{\max } t}
$$

for $0 \leq t \leq T^{\delta}$, and $\rho>0$ is the gap between the largest growth rate $\lambda_{\max }$ and the rest of $\operatorname{Re} \lambda_{i}(\mathbf{q})$ in (7), $e^{\mathfrak{M} t} \mathbf{w}_{0}(\mathbf{x})$ defined in (16) is the dominant part of the solution of the linearized system (5). 
Proof. Let $\mathbf{w}^{\delta}(\mathbf{x}, t)$ be the solutions to (4) with initial data $\mathbf{w}^{\delta}(\cdot, 0)=\delta \mathbf{w}_{0}$. Define

$$
\begin{gathered}
T^{*}=\sup \left\{t \mid\left\|\mathbf{w}^{\delta}(\cdot, t)-\delta e^{\mathfrak{L} t} \mathbf{w}_{0}\right\| \leq \frac{\hat{C}_{1}}{2} \delta e^{\lambda_{\max } t}\right\}, \\
T^{* *}=\sup \left\{t \mid\left\|\mathbf{w}^{\delta}(\cdot, t)\right\|_{H^{2}\left(\mathbb{T}^{d}\right)} \leq \frac{1}{\hat{C}_{2}} \min \left\{\frac{d_{1}}{4}, \frac{k d_{2}}{2}, \frac{3 d_{3}}{2}\right\}\right\} .
\end{gathered}
$$

From the definition of $T^{*}$ and Lemma 5 , for $\forall 0 \leq t \leq T^{*}$, we can obtain

$$
\left\|\mathbf{w}^{\delta}(\cdot, t)\right\| \leq \frac{3}{2} \hat{C}_{1} \delta e^{\lambda_{\max }}
$$

Furthermore, by Lemma 8 and the bootstrap argument, we possess

$$
\left\|\mathbf{w}^{\delta}(\cdot, t)\right\|_{H^{2}\left(\mathbb{T}^{d}\right)} \leq \sqrt{\hat{C}_{4}}\left\{\delta\left\|\mathbf{w}_{0}\right\|_{H^{2}\left(\mathbb{T}^{d}\right)}+\delta e^{\lambda_{\max } t}\right\}
$$

Applying Duhamel's principle, we know that the solution of (4)

$$
\mathbf{w}^{\delta}(\cdot, t)=\delta e^{\mathfrak{L} t} \mathbf{w}_{0}-\int_{0}^{t} e^{\mathfrak{L}(t-\tau)}\left[\chi \nabla\left(u_{1}^{\delta}(\tau) \nabla u_{2}^{\delta}(\tau)\right)+\xi \nabla\left(u_{1}^{\delta}(\tau) \nabla u_{3}^{\delta}(\tau)\right)+\mu u_{1}^{\delta}(\tau)\left(1+u_{1}^{\delta}(\tau)\right), 0,0\right] d \tau .
$$

It follows from Lemma 5, (40), (44) and Lemma 8 that for $0 \leq t \leq \min \left\{T^{\delta}, T^{*}, T^{* *}\right\}$,

$$
\left\|\mathbf{w}^{\delta}(\cdot, t)-\delta e^{\mathfrak{L} t} \mathbf{w}_{0}\right\| \leq \hat{C}_{1} \hat{C}_{5} \int_{0}^{t} e^{\lambda_{\max }(t-\tau)}\left\|\mathbf{w}^{\delta}(\tau)\right\|_{H^{2}\left(\mathbb{T}^{d}\right)}^{2} d \tau,
$$

where $\hat{C}_{5}=\max C_{9}^{2}\left\{\chi+\chi \frac{C_{4}}{C_{9}^{2}}+\xi+\xi \frac{C_{4}}{C_{9}^{2}}+\mu C_{1}\right\}$. By (66) and (68), we see that for $t \leq \min \left\{T^{\delta}, T^{*}, T^{* *}\right\}$,

$$
\left\|\mathbf{w}^{\delta}(\cdot, t)-\delta e^{\mathfrak{s} t} \mathbf{w}_{0}\right\| \leq \hat{C}_{1} \hat{C}_{4} \hat{C}_{5}\left\{\frac{\delta\left\|\mathbf{w}_{0}\right\|_{H^{2}}^{2}}{\lambda_{\max }}+\frac{\delta e^{\lambda_{\max } t}}{\lambda_{\max }}\right\} \delta e^{\lambda_{\max } t} .
$$

We now prove that if $\delta_{0}$ and $\theta$ are chosen such that

$$
\theta<\frac{1}{\hat{C}_{2} \hat{C}_{4}} \min \left\{\frac{\lambda_{\max }}{4}, \frac{d_{1}}{8}, \frac{k d_{2}}{4}, \frac{3 d_{3}}{4}\right\}
$$

and

$$
\sqrt{\hat{C}_{4}} \delta_{0}\left\|\mathbf{w}_{0}\right\|_{H^{2}\left(\mathbb{T}^{d}\right)} \leq \frac{1}{2 \hat{C}_{2}} \min \left\{\frac{d_{1}}{4}, \frac{k d_{2}}{2}, \frac{3 d_{3}}{2}\right\}
$$

as well as

$$
\hat{C}_{4} \hat{C}_{5} \frac{\delta_{0}\left\|\mathbf{w}_{0}\right\|_{H^{2}\left(\mathbb{T}^{d}\right)}^{2}}{\lambda_{\max }}<\frac{1}{4}
$$

then $T^{\delta}=\min \left\{T^{\delta}, T^{*}, T^{* *}\right\}$ for $\delta \leq \delta_{0}$.

If $T^{* *}$ is the smallest, we can let $t=T^{* *} \leq T^{\delta}$ in (67). By (70) and (71) we have

$$
\left\|\mathbf{w}^{\delta}\left(T^{* *}\right)\right\|_{H^{2}\left(\mathbb{T}^{d}\right)} \leq \sqrt{\hat{C}_{4}}\left\|\mathbf{w}_{0}^{\delta}\right\|_{H^{2}\left(\mathbb{T}^{d}\right)}+\sqrt{C_{4}} \theta<\frac{1}{\hat{C}_{2}} \min \left\{\frac{d_{1}}{4}, \frac{d_{2}}{4}, \frac{d_{3} K}{2}\right\}
$$

for $\delta$ sufficiently small and $\hat{C}_{4} \geq 1$, in contradiction to the definition of $T^{* *}$. On the other hand, if $T^{*}$ is the minimum, we can let $t=T^{*}$ in (67), so that

$$
\left\|\mathbf{w}^{\delta}\left(\cdot, T^{*}\right)-\delta e^{\mathfrak{L} T^{*}} \mathbf{w}_{0}\right\| \leq \hat{C}_{1} \hat{C}_{4} \hat{C}_{5}\left\{\frac{\delta\left\|\mathbf{w}_{0}\right\|_{H^{2}\left(\mathbb{T}^{d}\right)}^{2}}{\lambda_{\max }}+\frac{\theta}{\lambda_{\max }}\right\} \delta e^{\lambda_{\max } T^{*}}<\frac{\hat{C}_{1}}{2} \delta e^{\prime}
$$


for sufficiently small $\delta_{0}$ in (73) and $\hat{C}_{5} / \hat{C}_{2} \leq 1$. This again contradicts the definition of $T^{*}$. Therefore, the desired assertion follows. Finally, we prove the inequality (62). Notice by (14) that

$$
\begin{aligned}
& \left\|\mathbf{w}^{\delta}(\cdot, t)-\delta e^{\mathfrak{M} t} \mathbf{w}_{0}\right\| \leq\left\|\mathbf{w}^{\delta}(\cdot, t)-\delta e^{\mathfrak{L} t} \mathbf{w}_{0}\right\|+\left\|\delta \sum_{\mathbf{q} \in \Lambda_{R 1}} \sum_{i \in I \backslash I_{1}} w_{i}(\mathbf{q}) \mathbf{r}_{i}(\mathbf{q}) e^{\lambda_{i} t} e_{\mathbf{q}}(\mathbf{x})\right\| \\
& +\left\|\delta \sum_{\mathbf{q} \in \mathbb{N}_{R 1}^{d} \backslash \Lambda_{R 1}} \sum_{i \in I} w_{i}(\mathbf{q}) \mathbf{r}_{i}(\mathbf{q}) e^{\lambda_{i} t} e_{\mathbf{q}}(\mathbf{x})\right\| \\
& +\left\|\delta \sum_{\mathbf{q} \in \mathbb{N}_{R 2}^{d}}\left\{\left[w_{d}(\mathbf{q}) \mathbf{r}_{d}(\mathbf{q})+w_{d}^{\prime}(\mathbf{q})\left(\mathbf{r}_{d}^{\prime}(\mathbf{q})+\mathbf{r}_{d}(\mathbf{q}) t\right)\right] e^{\lambda_{d}(\mathbf{q}) t}+w_{s}(\mathbf{q}) \mathbf{r}_{s}(\mathbf{q}) e^{\lambda_{s}(\mathbf{q}) t}\right\} e_{\mathbf{q}}(\mathbf{x})\right\| \\
& +\left\|\delta \sum_{\mathbf{q} \in \mathbb{N}_{R 3}^{d}}\left[w(\mathbf{q}) \mathbf{r}(\mathbf{q})+w^{\prime}(\mathbf{q})\left(\mathbf{r}^{\prime}(\mathbf{q})+\mathbf{r}(\mathbf{q}) t\right)+w^{\prime \prime}(\mathbf{q})\left(\mathbf{r}^{\prime \prime}(\mathbf{q})+\mathbf{r}^{\prime}(\mathbf{q}) t+\mathbf{r}(\mathbf{q}) t^{2}\right)\right] e^{\lambda(\mathbf{q}) t} e_{\mathbf{q}}(\mathbf{x})\right\| \\
& +\left\|\delta \sum_{\mathbf{q} \in \Lambda_{C 1}} w_{r}(\mathbf{q}) \mathbf{r}_{r}(\mathbf{q}) e^{\lambda_{r}(\mathbf{q}) t} e_{\mathbf{q}}(\mathbf{x})\right\|+\| \delta \sum_{\mathbf{q} \in \Lambda_{C 2}}\left[w^{\operatorname{Re}}(\mathbf{q})\left(\operatorname{Rer}_{c}(\mathbf{q}) \cos \left[\left(\operatorname{Im} \lambda_{c}(\mathbf{q})\right) t\right]-\operatorname{Im} \mathbf{r}_{c}(\mathbf{q}) \sin \left[\left(\operatorname{Im} \lambda_{c}(\mathbf{q})\right) t\right]\right)\right. \\
& \left.+w^{\operatorname{Im}}(\mathbf{q})\left(\operatorname{Rer}_{c}(\mathbf{q}) \sin \left[\left(\operatorname{Im} \lambda_{c}(\mathbf{q})\right) t\right]+\operatorname{Im} \mathbf{r}_{c}(\mathbf{q}) \cos \left[\left(\operatorname{Im} \lambda_{c}(\mathbf{q})\right) t\right]\right)\right] e^{\left(\operatorname{Re} \lambda_{c}(\mathbf{q})\right) t} e_{\mathbf{q}}(\mathbf{x}) \| \\
& +\| \delta \sum_{\mathbf{q} \in \mathbb{N}_{C}^{d} \backslash \Lambda_{C 3}}\left\{\left[w^{\operatorname{Re}}(\mathbf{q})\left(\operatorname{Rer}_{c}(\mathbf{q}) \cos \left[\left(\operatorname{Im} \lambda_{c}(\mathbf{q})\right) t\right]-\operatorname{Im} \mathbf{r}_{c}(\mathbf{q}) \sin \left[\left(\operatorname{Im} \lambda_{c}(\mathbf{q})\right) t\right]\right)\right.\right. \\
& \left.\left.+w^{\operatorname{Im}}(\mathbf{q})\left(\operatorname{Rer}_{c}(\mathbf{q}) \sin \left[\left(\operatorname{Im} \lambda_{c}(\mathbf{q})\right) t\right]+\operatorname{Imr} \mathbf{r}_{c}(\mathbf{q}) \cos \left[\left(\operatorname{Im} \lambda_{c}(\mathbf{q})\right) t\right]\right)\right] e^{\left(\operatorname{Re} \lambda_{c}(\mathbf{q})\right) t}+w_{r}(\mathbf{q}) \mathbf{r}_{r}(\mathbf{q}) e^{\lambda_{r}(\mathbf{q}) t}\right\} e_{\mathbf{q}}(\mathbf{x}) \| \\
& :=\left\|\mathbf{w}^{\delta}(\cdot, t)-\delta e^{\mathfrak{L} t} \mathbf{w}_{0}\right\|+J_{6}+J_{7}+J_{8}+J_{9}+J_{10}+J_{11}+J_{12} .
\end{aligned}
$$

We next estimate each term $J_{i}(i=6,7,8, \cdots, 12)$ on the right-hand sides of (73). It is not difficult to know that there are finitely many values $\mathbf{q} \in \mathbb{N}^{d}$ satisfying $\operatorname{Re} \lambda_{i}(\mathbf{q})=\lambda_{\max }$ and $|\mathbf{q}|$ is bounded for each $\mathbf{q} \in \mathbb{N}^{d}$ max. For each $\mathbf{q} \in \mathbb{N}^{d}, q^{2}<N$ there exists a constant $C_{*}>0$ such that

$$
\begin{cases}\left|\mathbf{r}_{1}(\mathbf{q})\right|,\left|\mathbf{r}_{2}(\mathbf{q})\right|,\left|\mathbf{r}_{3}(\mathbf{q})\right| \leq C_{*,} & \mathbf{q} \in \mathbb{N}_{R 1}^{d} \\ \left|\mathbf{r}_{d}(\mathbf{q})\right|,\left|\mathbf{r}^{\prime}(\mathbf{q})\right|,\left|\mathbf{r}_{s}(\mathbf{q})\right| \leq C_{*,} & \mathbf{q} \in \mathbb{N}_{R 2}^{d} \\ |\mathbf{r}(\mathbf{q})|,\left|\mathbf{r}^{\prime}(\mathbf{q})\right|,\left|\mathbf{r}^{\prime \prime}(\mathbf{q})\right| \leq C_{*}, & \mathbf{q} \in \mathbb{N}_{R 3}^{d} \\ \left|\operatorname{Rer}_{c}(\mathbf{q})\right|,\left|\operatorname{Imr}_{c}(\mathbf{q})\right|,\left|\mathbf{r}_{r}(\mathbf{q})\right| \leq C_{*}, & \mathbf{q} \in \mathbb{N}_{C}^{d}\end{cases}
$$

By the similar method to prove (32), using (74) and (13) there exists a constant $C_{* *}>0$ such that

$$
\begin{cases}\left|w_{1}(\mathbf{q})\right|,\left|w_{2}(\mathbf{q})\right|,\left|w_{3}(\mathbf{q})\right| \leq C_{* *}\left|\mathbf{w}_{\mathbf{q}}\right|, & \mathbf{q} \in \mathbb{N}_{R 1}^{d}, \\ \left|w_{d}(\mathbf{q})\right|,\left|w_{d}^{\prime}(\mathbf{q})\right|,\left|w_{s}(\mathbf{q})\right| \leq C_{* *}\left|\mathbf{w}_{\mathbf{q}}\right|, & \mathbf{q} \in \mathbb{N}_{R 2^{\prime}}^{d} \\ |w(\mathbf{q})|,\left|w^{\prime}(\mathbf{q})\right|,\left|w^{\prime \prime}(\mathbf{q})\right| \leq C_{* *}\left|\mathbf{w}_{\mathbf{q}}\right|, & \mathbf{q} \in \mathbb{N}_{R 3^{\prime}}^{d} \\ \left|w^{\operatorname{Re}}(\mathbf{q})\right|,\left|w^{\operatorname{Im}}(\mathbf{q})\right|,\left|w_{r}(\mathbf{q})\right| \leq C_{* *}\left|\mathbf{w}_{\mathbf{q}}\right|, & \mathbf{q} \in \mathbb{N}_{C}^{d}\end{cases}
$$

and

$$
t e^{\lambda_{d}(\mathbf{q}) t} \leq C_{* *}, \text { for } \mathbf{q} \in \mathbb{N}_{R 2}^{d}, t e^{\lambda(\mathbf{q}) t}, t^{2} e^{\lambda(\mathbf{q}) t} \leq C_{* *}, \text { for } \mathbf{q} \in \mathbb{N}_{R 3}^{d} .
$$

By (29), (32),(74), (75) and $\left\|\mathbf{w}_{0}\right\|=1$, there exists a constant $\hat{C}_{6}>0$ such that

$$
J_{7}^{2} \leq \delta^{2} \hat{C}_{6}^{2} e^{2\left(\lambda_{\max }-\rho\right) t}\left(\frac{\pi}{2}\right)^{d} \sum_{\mathbf{q} \in \Lambda_{R 1}}\left|\mathbf{w}_{\mathbf{q}}\right|^{2} \leq \delta^{2} \hat{C}_{6}^{2} e^{2\left(\lambda_{\max }-\rho\right) t}\left\|\mathbf{w}_{0}\right\|^{2} \leq \delta^{2} \hat{C}_{6}^{2} e^{2\left(\lambda_{\max }-\rho\right) t},
$$

that is,

$$
J_{6} \leq \delta \hat{C}_{6} e^{\left(\lambda_{\max }-\rho\right) t} .
$$

Moreover,

$$
J_{7} \leq \delta e^{\left(\lambda_{\max }-\rho\right) t} .
$$


Similarly, there exists a constant $\hat{C}_{7}>0$ such that

$$
J_{i} \leq \delta \hat{C}_{7} e^{\left(\lambda_{\max }-\rho\right) t}, i=8, \cdots, 12 .
$$

Substituting (69), (77)-(81) into (73) yields

$$
\begin{aligned}
\left\|\mathbf{w}^{\delta}(\cdot, t)-\delta e^{\mathfrak{M} t} \mathbf{w}_{0}\right\| & \leq \hat{C}_{1} \hat{C}_{4} \hat{C}_{5}\left\{\frac{\delta\left\|\mathbf{w}_{0}\right\|_{H^{2}}^{2}}{\lambda_{\max }}+\frac{\delta e^{\lambda_{\max } t}}{\lambda_{\max }}\right\} \delta e^{\lambda_{\max } t}+\hat{C}_{6} \delta e^{\left(\lambda_{\max }-\rho\right) t}+\delta e^{\left(\lambda_{\max }-\rho\right) t}+5 \hat{C}_{7} \delta e^{\left(\lambda_{\max }-\rho\right) t} \\
& \leq\left\{\left(1+\hat{C}_{6}+5 \hat{C}_{7}\right) e^{-\rho t}+\frac{\hat{C}_{1} \hat{C}_{4} \hat{C}_{5}}{\lambda_{\max }}\left(\delta\left\|\mathbf{w}_{0}\right\|_{H^{2}\left(\mathbb{T}^{d}\right)}^{2}+\delta e^{\lambda_{\max } t}\right)\right\} \delta e^{\lambda_{\max } t} \\
& \leq \hat{C}\left\{e^{-\rho t}+\delta\left\|\mathbf{w}_{0}\right\|_{H^{2}\left(\mathbb{T}^{d}\right)}^{2}+\delta e^{\lambda_{\max } t}\right\} \delta e^{\lambda_{\max } t}, \forall 0 \leq t \leq T^{\delta},
\end{aligned}
$$

where $\hat{C}:=\max \left\{1+\hat{C}_{6}+5 \hat{C}_{7}, \frac{\hat{C}_{1} \hat{C}_{4} \hat{C}_{5}}{\lambda_{\max }}\right\}$ and thereby completes the proof.

Corollary 1. (Nonlinear instability). Let the conditions $\left(\mathbf{H}_{1}\right),\left(\mathbf{H}_{2}\right)$ and $\left(\mathbf{H}_{3}\right)$ are holds. Then the positive constant equilibrium point $\mathbf{w}_{c}$ of the problem (1) is nonlinearly unstable in the sense of the $L^{2}$-norm.

Proof. Notice that $\mathbf{L}_{\mathbf{q}_{0}}$ has an eigenvalue $\operatorname{Re} \lambda_{\mathbf{q}_{0}}=\lambda_{\max }$, if there exists $\mathbf{q}_{0}=\left(q_{01}, \ldots, q_{0 d}\right) \in \mathbb{N}^{d}$ max , and denote the corresponding eigenvector by $\mathbf{r}_{\mathbf{q}_{0}}$. Assume

$$
\mathbf{w}_{0}(\mathbf{x})=\kappa \frac{\mathbf{r}\left(\mathbf{q}_{0}\right)}{\left|\mathbf{r}\left(\mathbf{q}_{0}\right)\right|} e_{\mathbf{q}_{0}}(\mathbf{x})
$$

with $\kappa=1 /\left\|e_{\mathbf{q}_{0}}\right\|=\sqrt{(2 / \pi)^{d}}$ so that $\left\|\mathbf{w}_{0}(x)\right\|=1$. In addition, if $t=T^{\delta}$ then for $\delta$ sufficiently small, we require

$$
\left\{\begin{array}{l}
\delta\left\|\mathbf{w}_{0}(\mathbf{x})\right\|_{H^{2}\left(\mathbb{T}^{d}\right)}^{2} \leq \frac{1}{4 \hat{C}^{\prime}} \\
e^{-\rho T^{\delta}}=\left(\frac{\delta}{\theta}\right)^{\frac{\rho}{\max }}<\frac{1}{8 \hat{C}^{\prime}} \\
\theta=\delta e^{\lambda_{\max } T^{\delta}}<\frac{1}{8 \hat{C}} .
\end{array}\right.
$$

It follows from Theorem 2 and (80) that

$$
\left\|\delta e^{\mathfrak{M} T^{\delta}} \mathbf{w}_{0}\right\|-\left\|\mathbf{w}^{\delta}\left(\cdot, T^{\delta}\right)\right\| \leq\left\|\mathbf{w}^{\delta}\left(\cdot, T^{\delta}\right)-\delta e^{\mathfrak{M} T^{\delta}} \mathbf{w}_{0}\right\| \leq \hat{C}\left\{e^{-\rho T^{\delta}}+\delta\left\|\mathbf{w}_{0}\right\|_{H^{2}\left(\mathbb{T}^{d}\right)}^{2}+\theta\right\} \theta<\frac{1}{2} \theta .
$$

Notice that the dominant part of the solution of the linearized system (5) satisfies

$$
\left\|\delta e^{\mathfrak{M} T^{\delta}} \mathbf{w}_{0}\right\|=\left\|\delta e^{\lambda_{\max } T^{\delta}} \mathbf{w}_{0}\right\|=\delta e^{\lambda_{\max } T^{\delta}}=\theta .
$$

By (81) and (82), we deduce that

$$
\left\|\mathbf{w}^{\delta}\left(\cdot, T^{\delta}\right)\right\|>\frac{1}{2} \theta>0
$$

Author Contributions: All authors contributed equally to the writing of this paper. All authors read and approved the final manuscript.

Conflicts of Interest: "The authors declare no conflict of interest."

\section{References}

[1] Horstmann, D. (2003). From 1970 until present: the Keller-Segel model in chemotaxis and its consequences. Jahresbericht der Deutschen Mathematiker-Vereinigung 105(3), 103-165.

[2] Jäger, W., \& Luckhaus, S. (1992). On explosions of solutions to a system of partial differential equations modelling chemotaxis. Transactions of the american mathematical society, 329(2), 819-824. 
[3] Winkler, M. (2010). Aggregation vs. global diffusive behavior in the higher-dimensional Keller-Segel model. Journal of Differential Equations, 248(12), 2889-2905.

[4] Winkler, M. (2013). Finite-time blow-up in the higher-dimensional parabolicÜparabolic Keller-Segel system. Journal de Mathématiques Pures et Appliquées, 100(5), 748-767.

[5] Keller, E. F., \& Segel, L. A. (1970). Initiation of slime mold aggregation viewed as an instability. Journal of theoretical biology, 26(3), 399-415.

[6] Osaki, K., \& Yagi, A. (1999). Structure of the stationary solution to Keller-Segel equation in one dimension (Nonlinear Evolution Equations and Applications). Journal of Mathematical Understanding and Analysis, (1105), 1-9.

[7] Horstmann, D., \& Wang, G. (2001). Blow-up in a chemotaxis model without symmetry assumptions. European Journal of Applied Mathematics, 12(2), 159-177.

[8] Herrero, M. A., \& Velázquez, J. J. (1996). Singularity patterns in a chemotaxis model. Mathematische Annalen, 306(1), 583-623.

[9] Winkler, M. (2011). Blow-up in a higher-dimensional chemotaxis system despite logistic growth restriction. Journal of Mathematical Analysis and Applications, 384(2), 261-272.

[10] Horstmann, D. (2004). From 1970 until present: the Keller-Segel model in chemotaxis and its consequences. IIJahresbericht der Deutschen Mathematiker-Vereinigung 106, 51-69.

[11] Horstmann, D. (2011). Generalizing the Keller-Segel model: Lyapunov functionals, steady state analysis, and blow-up results for multi-species chemotaxis models in the presence of attraction and repulsion between competitive interacting species. Journal of nonlinear science, 21(2), 231-270.

[12] Tello, J. I., \& Winkler, M. (2007). A chemotaxis system with logistic source. Communications in Partial Differential Equations, 32(6), 849-877.

[13] Aida, M., \& Yagi, A. (2004). Target pattern solutions for chemotaxis-growth system. Scientiae Mathematicae Japonicae, 59(3), 577-590.

[14] Kurata, N. (2008). Bifurcation phenomena of pattern solution to Mimura-Tsujikawa model in one dimension, GAKUTO International Series. Mathematical Sciences and Applications, 29, 265-278.

[15] Painter, K. J., \& Hillen, T. (2011). Spatio-temporal chaos in a chemotaxis model. Physica D: Nonlinear Phenomena, 240(4-5), 363-375.

[16] Okuda, T., \& Osaki, K. (2011). Bifurcation of hexagonal patterns in a chemotaxis-diffusion-growth system. Nonlinear Analysis: Real World Applications, 12(6), 3294-3305.

[17] Kuto, K., Osaki, K., Sakurai, T., \& Tsujikawa, T. (2012). Spatial pattern formation in a chemotaxis-diffusion-growth model. Physica D: Nonlinear Phenomena, 241(19), 1629-1639.

[18] Banerjee, S., Misra, A. P., \& Rondoni, L. (2012). Spatiotemporal evolution in a (2+1)-dimensional chemotaxis model. Physica A: Statistical Mechanics and its Applications, 391(1-2), 107-112.

[19] Guo, Y., \& Hwang, H. J. (2010). Pattern formation (I): the Keller-Segel model. Journal of Differential Equations, 249(7), 1519-1530.

[20] Fu, S., \& Liu, J. (2013). Spatial pattern formation in the Keller-Segel model with a logistic source. Computers $\mathcal{E}$ Mathematics with Applications, 66(3), 403-417.

[21] Zhang, T., \& Zang, H. (2014). Delay-induced Turing instability in reaction-diffusion equations. Physical Review E, 90(5), 052908.

[22] Zhang, T., Xing, Y., Zang, H., \& Han, M. (2014). Spatio-temporal dynamics of a reaction-diffusion system for a predator-prey model with hyperbolic mortality. Nonlinear Dynamics, 78(1), 265-277.

[23] Okubo, A. (1980). Diffusion and ecological problems: mathematical models, Biomathematics. Springer-Verlag, Berlin Heidelberg.

[24] Segel, L. A., \& Jackson, J. L. (1972). Dissipative structure: an explanation and an ecological example. Journal of theoretical biology, 37(3), 545-559.

[25] Peng, Y., \& Zhang, T. (2014). Stability and Hopf bifurcation analysis of a gene expression model with diffusion and time delay. In Abstract and Applied Analysis (Vol. 2014). Hindawi. Article ID 738682.

[26] Tang, X., \& Song, Y. (2015). Cross-diffusion induced spatiotemporal patterns in a predator-prey model with herd behavior. Nonlinear Analysis: Real World Applications, 24, 36-49.

[27] Almirantis, Y., \& Papageorgiou, S. (1991). Cross-diffusion effects on chemical and biological pattern formation. Journal of theoretical biology, 151(3), 289-311.

[28] Song, Y., Zhang, T., \& Peng, Y. (2016). TuringÜHopf bifurcation in the reaction-diffusion equations and its applications. Communications in Nonlinear Science and Numerical Simulation, 33, 229-258.

[29] Guo, Y., \& Strauss, W. A. (1995). Instability of periodic BGK equilibria. Communications on Pure and Applied Mathematics, 48(8), 861-894. 
[30] Hu, Z., Teng, Z., \& Jiang, H. (2012). Stability analysis in a class of discrete SIRS epidemic models. Nonlinear Analysis: Real World Applications, 13(5), 2017-2033.

[31] Fan, S. (1989). A new extracting formula and a new distinguishing means on the one variable cubic equation. Nat. Sci. J. Hainan Teach. Coll, 2(2), 91-98.

(c) 2020 by the authors; licensee PSRP, Lahore, Pakistan. This article is an open access article distributed under the terms and conditions of the Creative Commons Attribution (CC-BY) license (http://creativecommons.org/licenses/by/4.0/). 